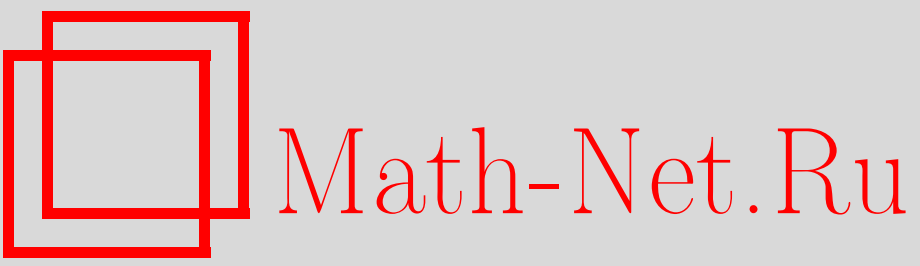

И. Д. Чуешов, Теория функционалов, однозначно определяющих асимптотическую динамику бесконечномерных диссипативных систем, УМН, 1998, том 53, выпуск 4, 77-124

DOI: https://doi.org/10.4213/rm57

Использование Общероссийского математического портала Math-Net.Ru подразумевает, что вы прочитали и согласны с пользовательским соглашением

http://www.mathnet.ru/rus/agreement

Параметры загрузки:

IP: 54.147 .182 .235

26 апреля 2023 г., 16:09:10 


\title{
ТЕОРИЯ ФУНКЦИОНАЛОВ, ОДНОЗНАЧНО ОПРЕДЕЛЯЮШИХ АСИМПТОТИЧЕСКУЮ ДИНАМИКУ БЕСКОНЕЧНОМЕРНЫХ ДИССИПАТИВНЫХ СИСТЕМ
}

\author{
И. Д. ЧуЕшов \\ СОДЕРЖАНИЕ
}

Введение . . . . . . .

$\S 1$. Понятие множества определяющих функционалов ................. 79

$\S 2$. Дефект полноты множества функционалов ..................... 87

§3. Оценки дефекта полноты в соболевских пространствах .......... 93

$\S 4$. Определяющие функционалы для полулинейных параболических уравнений …........................................... 101

§5. Определяющие функционалы для уравнений второго порядка по вре-

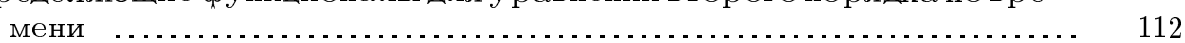

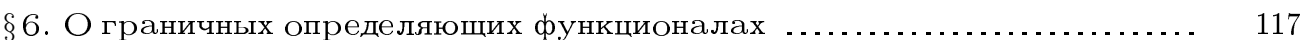

Список литературы .............................................. 122

\section{Введение}

Основньми объектами, возникаюшими при исследовании асимптотического поведения бесконечномерных диссипативных динамических систем, являются аттракторы. Их изучение позволяет ответить на ряд вопросов принципиального характера о свойствах предельных режимов, которые могут возникнуть в рассматриваемой системе. В настоящее время выработаны довольно универсальные подходы и методы, позволяюшие доказьвать существование и конечномерность глобальных аттракторов для широкого класса динамических систем, порождаемых нелинейными уравнениями в частных производных (см., например, [1]-[5] и ссылки, приведенные в этих работах). Однако детально исследовать структуру аттрактора удается лишь для весьма ограниченного класса задач. В этой связи важньм является вопрос об отыскании минимальных (или близких к минимальньм) множеств естественных параметров задачи, которые однозначно определяют асимптотическое поведение системы. Впервые данньй вопрос рассматривался для двумерной системы Навье-Стокса в работах [6] и [7], где было показано, что асимптотическое поведение решений полностью определяется динамикой первых $N$ мод Фурье, если $N$ достаточно велико. После этих работ подобные результаты были получены для других параметров и уравнений (см., например, 
[8]-[21] и ссылки, приведенные в этих работах, а также в обзоре [4]). Были введены понятия определяюших узлов [8]-[10] и определяюших локальных объемных средних [12], [13]. Обсуждался вопрос о связи проблемы существования конечного числа определяющих параметров с некоторыми задачами теории интерполящии [17]-[21].

В данной статье развивается общая теория определяюших функционалов, позволяюшая, во-первых, охватить все указанные выше результаты с единой точки зрения, а во-вторых, предложить достаточно простые условия, при которых множество линейных функционалов на фазовом пространстве однозначно определяет своими значениями на траекториях системы ее асимптотическое поведение. Предлагаемьй здесь подход опирается на понятие дефекта полноты множества функционалов и привлекает некоторые идеи и результаты теории аппроксимаций бесконечномерных пространств.

Кратко опишем структуру работы.

В $\S 1$ вводятся и обсуждаются двапонятия определяюших функционалов. Они представляют собой далеко идушие расширения понятий определяюших мод, введенных соответственно Фояшем и Проди [6] и Ладыженской [7]. Приведено несколько достаточно простых утверждений общего характера о существовании конечных семейств определяющих функционалов. Утверждения подобного типа, с одной стороны, неявно привлекались ранее при изучении конкретных задач, а с другой, они дают представление о том, какие именно свойства динамической системы гарантируют конечность числа определяюших элементов.

В $\S 2$ вводится понятие дефекта полноты $\varepsilon \mathscr{L}(V, H)$ системы функционалов $\mathscr{L}$ относительно пары пространств $V \subset H$ и изучаются его свойства. При этом оказалось, что для широкого класса диссипативных систем условие малости дефекта $\varepsilon \mathscr{L}(V, H)$ является достаточным для того, чтобы множество $\mathscr{L}$ функционалов на $V$ было определяюшим. Установлена также связь величины $\varepsilon \mathscr{L}(V, H)$ с поперечником вложения Колмогорова пространства $V$ в $H$ и предложены довольно простые процедуры, позволяюшие эффективно оценивать дефекты полноты. Результаты этого параграфа используются в $\S 3$ для вычисления оценок дефектов полноты для двух, важных с точки зрения приложений, семейств функционалов на соболевских пространствах. Речь идет об обобщенных локальных объемных средних и о наборе $\delta$-функций (узлов). Полученные оценки по числу $(N)$ функционалов имеют тот же порядок, что и соответствующий $N$-поперечник Колмогорова, и являются неулучшаемыми. Результаты $\S 3$ позволяют, в частности, в последуюших разделах установить существование конечных семейств определяющих функционалов для ряда диссипативных систем, порождаемых нелинейными уравнениями в частных производных, а также получить наилучшие (в определенном смысле) оценки числа этих функционалов.

Оставшиеся разделы статьи (§4-§6) посвяшены приложениям. В 44 рассматриваются полулинейные параболические уравнения. В качестве основных примеров обсуждаются двумерная система Навье-Стокса и система уравнений Ходжкина-Хаксли, описываюшая передачу нервных импульсов. Оказалось, что в этих примерах определяюшие функционалы можно также задавать и на пространствах, не совпадающих с фазовым (и не содержаших его внутри себя). Кроме того, на примере системы Ходжкина-Хаксли продемонстрирована роль регулярности решений по Жевре при построении двухточечного множества определяющих узлов. Построению определяюших функционалов для нелинейных уравнений второго порядка по времени посвящен $\S 5$. Здесь обсуждается также случай уравнений с запаздыванием. В качестве примера 
рассматривается задача о нелинейных колебаниях упругой пологой оболочки в потенциальном линеаризованном потоке газа. В заключительном $\S 6$ показано, что для некоторых начально-краевых задач с нелинейными граничными условиями определяющие функционалы можно задавать лишш на гранище области.

\section{§1. Понятие множества определяющих функционалов}

Пусть в вещественном реффлексивном банаховом пространстве $H$ задано некоторое неавтономное дифференциальное уравнение:

$$
\frac{d u}{d t}=F(u, t), \quad t>0,\left.\quad u\right|_{t=0}=u_{0}
$$

Предположим, что $\mathscr{W}$ - некоторьй класс решений задачи (1.1), определенных на полуоси $\mathbb{R}_{+} \equiv\{t: t \geqslant 0\}$ и таких, что для каждого $u(t) \in \mathscr{W}$ сушествует момент времени $t_{0}>0$ такой, что

$$
u(t) \in C\left(t_{0},+\infty ; H\right) \cap L_{\mathrm{loc}}^{2}\left(t_{0},+\infty ; V\right),
$$

где $V$ - некоторое рефлексивное банахово пространство, непрерьвно вложенное в $H$. Здесь и в дальнейшем $C(a, b ; X)$ - пространство сильно непрерывных функций на $[a, b]$ со значениями в $X$, обозначение $L_{\text {loc }}^{2}(a, b ; X)$ имеет аналогичный смысл. Ниже используются также обозначения $\|\cdot\|_{H}$ и $\|\cdot\|_{V}$ для норм в пространствах $H$ и $V$, $\|\cdot\|_{H} \leqslant\|\cdot\|_{V}$.

В основу приведенного ниже определения положено свойство, установленное в [6] для мод Фурье решений двумерной системы Навье-Стокса с периодическими граничными условиями.

ОПРЕДЕЛЕНИЕ 1.1. Пусть $\mathscr{L}=\left\{l_{j}: j=1, \ldots, N\right\}$ - множество линейных непрерывных функционалов на $V$. Тогда $\mathscr{L}$ назьвается множеством асимптотически $(V, H, \mathscr{W})$-определяющих функиионалов (или элементов) задачи (1.1), если для любой пары ее решений $u, v \in \mathscr{W}$ из условия

$$
\lim _{t \rightarrow \infty} \int_{t}^{t+1}\left|l_{j}(u(\tau))-l_{j}(v(\tau))\right|^{2} d \tau=0 \quad \text { для } j=1, \ldots, N
$$

вытекает, что

$$
\lim _{t \rightarrow \infty}\|u(t)-v(t)\|_{H}=0
$$

В основе всех известных к настоящему моменту утверждений о существовании конечных множеств асимптотически определяющих функционалов фактически лежит следующее утверждение. 
Теорема 1.1. Пусть $\mathscr{L}=\left\{l_{j}: j=1, \ldots, N\right\}$ - некоторая совокупность линейных непрерывных функционалов на $V$. Предположим, что на $H \times \mathbb{R}_{+}$существует непрерывная функция $\mathcal{V}(u, t)$ со значениями в $\mathbb{R}_{+}$, обладающая свойствами:

а) найдутся положительнье числа $\alpha$ и $\sigma$ такие, что

$$
\mathscr{V}(u, t) \geqslant \alpha \cdot\|u\|^{\sigma} \quad \text { для всех } \quad u \in H, \quad t \in \mathbb{R}_{+} ;
$$

б) для любой пары решений $u(t), v(t) \in \mathscr{W}$ задачи (1.1) найдутся момент времени $t_{0}>0$, локально интегрируемая на интервале $\left[t_{0}, \infty\right)$ функиия $\psi(t)$ со свойствами

$$
\gamma_{\psi}^{+} \equiv \underset{t \rightarrow \infty}{\lim } \int_{t}^{t+a} \psi(\tau) d \tau>0
$$

$u$

$$
\Gamma_{\psi}^{+} \equiv \varlimsup_{t \rightarrow \infty} \int_{t}^{t+a} \max \{0,-\psi(\tau)\} d \tau<\infty
$$

при некотором $a>0$ и положительная константа $C$ такие, что для всех $t \geqslant s \geqslant t_{0}$ выполнено соотношение

$$
\begin{aligned}
\mathscr{V}(u(t)- & v(t), t)+\int_{s}^{t} \psi(\tau) \cdot \mathscr{V}(u(\tau)-v(\tau), \tau) d \tau \\
& \leqslant \mathscr{V}(u(s)-v(s), s)+C \cdot \int_{s}^{t} \max _{j=1, \ldots, N}\left|l_{j}(u(\tau))-l_{j}(v(\tau))\right|^{2} d \tau
\end{aligned}
$$

Тогда $\mathscr{L}$ является множеством асимптотически $(V, H, \mathscr{W})$-определяющих функчионалов задачи (1.1).

Очевидно, что доказательство этой теоремы вытекает из следующего варианта леммы Гронуолла.

Лемма 1.1. Пусть $\psi(t)$ и $g(t)$ - локально интегрируемые на интервале $\left[t_{0}, \infty\right)$ функиии такие, что для $\psi(t)$ выполнены соотношения (1.6) u (1.7), а g(t) неотричательна и обладает свойством

$$
\lim _{t \rightarrow \infty} \int_{t}^{t+a} g(\tau) d \tau=0, \quad a>0 .
$$

Предположим, что $w(t)$ - неотрицательная непрерывная функиия, удовлетворяющая неравенству

$$
w(t)+\int_{s}^{t} \psi(\tau) \cdot w(\tau) d \tau \leqslant w(s)+\int_{s}^{t} g(\tau) d \tau
$$

для всех $t \geqslant s \geqslant t_{0}$. Тогда $w(t) \rightarrow 0$ при $t \rightarrow \infty$.

Отметим, что данный вариант леммы Гронуолла использовался многими авторами (см., например, [2], [8], [13], [14], [22]). Приведенная здесь формулировка фактически совпадает с утверждением, содержашимся в [13]. 
ДокАЗАТЕльство леммы 1.1. Укажем основные этапы рассуждений (детали см. в [13] или [22]). Из (1.10) вытекает, что

$$
w(t) \leqslant w(s) \cdot \exp \left\{-\int_{s}^{t} \psi(\tau) d \tau\right\}+\int_{s}^{t} g(\tau) \cdot \exp \left\{-\int_{\tau}^{t} \psi(\sigma) d \sigma\right\} d \tau
$$

для всех $t \geqslant s \geqslant t_{0}$. Используя свойства $(1.6),(1.7)$, легко обнаружить, что сушествуют числа $t^{*} \geqslant t_{0}, a_{0}>0, b_{0}>0$ такие, что

$$
\int_{s}^{t} \psi(\tau) d \tau \geqslant a_{0} \cdot(t-s)-b_{0}, \quad t \geqslant s \geqslant t^{*}
$$

Поэтому из (1.11) вытекает, что

$$
\varlimsup_{t \rightarrow \infty} w(t) \leqslant C \cdot \varlimsup_{t \rightarrow \infty} \int_{s}^{t} g(\tau) \cdot \exp \left\{-a_{0} \cdot(t-\tau)\right\} d \tau .
$$

Далее, разбивая интеграл в правой части этого неравенства в сумму интегралов по интервалам длины $a$, как и в [13], [22], можно показать, что

$$
\varlimsup_{t \rightarrow \infty} w(t) \leqslant C \cdot \sup _{\sigma \geqslant s} \int_{\sigma}^{\sigma+a} g(\tau) d \tau .
$$

Отсюда вытекает утверждение леммы 1.1 .

В том случае, когда задача (1.1) представляет собой задачу Коши для квазилинейного уравнения в частных производных, в качестве функции $\mathscr{V}(u, t)$ при доказательстве существования конечного множества асимптотически определяюших элементов обычно фигурирует некоторая норма фазового пространства $Н$. Например, для параболических задач часто используется следующее утверждение, вытекающее из теоремы 1.1.

СлЕДСТВИЕ 1.1. Пусть $V$ и чем $V$ непрерывно вложено в $H$. Предположим, что для любой пары решений $u(t), v(t) \in \mathscr{W}$ задачи (1.1) найдется момент времени $t_{0}>0$ такой, что для всех $t \geqslant s \geqslant t_{0}$ вьполнено соотношение

$$
\begin{aligned}
& \|u(t)-v(t)\|_{H}^{2}+\nu \int_{s}^{t}\|u(\tau)-v(\tau)\|_{V}^{2} d \tau \\
& \quad \leqslant\|u(s)-v(s)\|_{H}^{2}+\int_{s}^{t} \phi(\tau) \cdot\|u(\tau)-v(\tau)\|_{H}^{2} d \tau .
\end{aligned}
$$

Здесь $\nu>0$, а положительная локально интегрируемая на интервале $\left[t_{0}, \infty\right)$ функция $\phi(t)$ удовлетворяет условию

$$
\varlimsup_{t \rightarrow \infty} \frac{1}{a} \int_{t}^{t+a} \phi(\tau) d \tau \leqslant R
$$

для некоторого а $>0$, где $R>0$ - некоторая константа, не зависящая от $u(t)$ u $v(t)$. Пусть $\mathscr{L}=\left\{l_{j}: j=1, \ldots, N\right\}$ - некоторая совокупность линейных непрерывных функиионалов на $V$, обладающая свойством

$$
\|w\|_{H} \leqslant C_{\mathscr{L}} \cdot \max _{j=1, \ldots, N}\left|l_{j}(w)\right|+\varepsilon \mathscr{L} \cdot\|w\|_{V}
$$

для любого $w \in V$, где $C_{\mathscr{L}}$ и $\varepsilon_{\mathscr{L}}$ - положительные константы. Тогда $\mathscr{L}$ является множеством асимптотически $(V, H, \mathscr{W})$-определяющих функиионалов задачи (1.1) при условии, что в⿻्L $<\sqrt{\nu / R}$. 
ДокАЗАТЕЛЬСТво. Из (1.15) вытекает, что

$$
\|w\|_{V}^{2} \geqslant(1+\delta)^{-1} \cdot \varepsilon_{\mathscr{L}}^{-2} \cdot\|w\|_{H}^{2}-C_{\mathscr{L}, \delta} \cdot \max _{j=1, \ldots, N}\left|l_{j}(w)\right|^{2}
$$

для любого $\delta>0$. Поэтому из (1.13) имеем, что

$$
\begin{aligned}
& \|u(t)-v(t)\|_{H}^{2}+\int_{s}^{t} \psi(\tau) \cdot\|u(\tau)-v(\tau)\|_{H}^{2} d \tau \\
& \quad \leqslant\|u(s)-v(s)\|_{H}^{2}+\nu C_{\mathscr{L}, \delta} \cdot \int_{s}^{t} \max _{j=1, \ldots, N}\left|l_{j}(u(\tau)-v(\tau))\right|^{2} d \tau,
\end{aligned}
$$

где $\psi(t)=\nu(1+\delta)^{-1} \varepsilon_{\mathscr{L}}^{-2}-\phi(t)$. Используя (1.14) и применяя теорему $1.1 \mathrm{c} \mathscr{V}(u, t)=$ $\|u\|^{2}$, получаем утверждение следствия 1.1.

Возможны и другие подходы к введению понятия определяюших функционалов. Приведенное ниже определение представляет собой распространение на общую ситуацию установленного О. А. Ладыженской [7] свойства траекторий, лежаших в глобальном аттракторе двумерной системы уравнений Навье-Стокса.

ОПРЕДЕЛЕниЕ 1.2. Пусть $\overline{\mathscr{W}}$ - класс решений задачи (1.1), определенных на всей вещественной оси $\mathbb{R}$, такой, что $\overline{\mathscr{W}} \subset L_{\mathrm{loc}}^{2}(-\infty,+\infty ; V)$. Совокупность $\mathscr{L}=\left\{l_{j}\right.$ : $j=1, \ldots, N\}$ линейных непрерьвных функщионалов на $V$ назьвается множеством $(V, \overline{\mathscr{W}})$-определяющих функиионалов (или элементов) задачи (1.1), если для любой пары ее решений $u, v \in \overline{\mathscr{W}}$ из условия

$$
l_{j}(u(t))=l_{j}(v(t)) \text { для почти всех } t \in \mathbb{R} \text { и } j=1, \ldots, N
$$

вытекает, что $u(t) \equiv v(t)$.

Легко может быть установлен следующий аналог теоремы 1.1.

Теорема 1.2. Пусть $\mathscr{L}=\left\{l_{j}: j=1, \ldots, N\right\}$ - некоторая совокупность линейных непрерывных функиионалов на $V$. Пусть $\overline{\mathscr{W}}-$ класс решений задачи (1.1), определенных на всей вещественной оси $\mathbb{R}$, такой, что

$$
\overline{\mathscr{W}} \subset C(-\infty,+\infty ; H) \cap L_{\mathrm{loc}}^{2}(-\infty,+\infty ; V) .
$$

Предположим, что на $H \times \mathbb{R}$ существует непрерьвная функция $\mathscr{V}(u, t)$ со значениями в $\mathbb{R}$, обладающая свойствами:

а) найдутся положительные числа $\alpha$ и $\sigma$ такие, что

$$
\mathscr{V}(u, t) \geqslant \alpha \cdot\|u\|^{\sigma} \quad \text { для всех } \quad u \in H, \quad t \in \mathbb{R}
$$

б) для любых $u(t), v(t) \in \overline{\mathscr{W}}$ выполнено соотношение

$$
\sup _{t \in \mathbb{R}} \mathscr{V}(u(t)-v(t), t)<\infty
$$


в) для любой парь решений $u(t), v(t) \in \overline{\mathscr{W}}$ задачи (1.1) найдутся локально интегрируемая на оси $\mathbb{R}$ функция $\psi(t)$ со свойствами

$$
\begin{aligned}
& \gamma_{\bar{\psi}}^{-} \equiv \underset{t \rightarrow-\infty}{\lim } \int_{t}^{t+a} \psi(\tau) d \tau>0, \\
& \Gamma_{\psi}^{-} \equiv \varlimsup_{t \rightarrow-\infty} \int_{t}^{t+a} \max \{0,-\psi(\tau)\} d \tau<\infty
\end{aligned}
$$

при некотором $a>0$ и положительная константа $C$ такие, что для всех $t \geqslant s$ выполнено соотношение (1.8).

Тогда $\mathscr{L}$ является множеством $(V, \overline{\mathscr{W}})$-определяющих функиионалов задачи (1.1).

ДокАЗАТЕЛЬство. Из (1.18), (1.8) и (1.11) вытекает, что функция $w(t)=$ $\mathscr{V}(u(t)-v(t), t)$ удовлетворяет неравенству

$$
w(t) \leqslant w(s) \cdot \exp \left\{-\int_{s}^{t} \psi(\tau) d \tau\right\}
$$

для всех $t \geqslant s$. Используя свойства (1.22), (1.23), легко обнаружить, что сушествуют числа $s^{*}, a_{0}>0, b_{0}>0$ такие, что

$$
\int_{s_{1}}^{s_{2}} \psi(\tau) d \tau \geqslant a_{0} \cdot\left(s_{2}-s_{1}\right)-b_{0}, \quad s_{1} \leqslant s_{2} \leqslant s^{*} .
$$

Это соотношение и свойство ограниченности (1.21) позволяют при фиксированном $t$ совершить в (1.24) предельный переход $s \rightarrow-\infty$ и получить искомое утверждение.

Так же, как и ранее, выбирая в теореме $1.2 \mathscr{V}(u, t)=\|u\|^{2}$, получаем следуюшее утверждение.

СЛЕДСТВИЕ 1.2. Пусть $V$ и чем $V$ непрерывно вложсено в $H$, и пусть $\overline{\mathscr{W}}$ - класс решений задачи (1.1), определенных на всей вещественной оси $\mathbb{R}$, обладающий свойством (1.19) и такой, чтоо

$$
\sup _{t \in \mathbb{R}}\|u(t)\|_{H}<\infty \quad \text { для } \quad \text { всех } \quad u(t) \in \overline{\mathscr{W}} .
$$

Предположим, что для любих $u(t), v(t) \in \overline{\mathscr{W}} u$ для всех вещественных $t \geqslant s$ выполняется соотношение (1.13), где $\nu>0$, а положительная локально интегрируемая на оси $\mathbb{R}$ функция $\phi(t)$ удовлетворяет условию

$$
\varlimsup_{s \rightarrow-\infty} \frac{1}{a} \int_{s}^{s+a} \phi(\tau) d \tau \leqslant R
$$

для некоторого а $>0$, где $R>0$ - некоторая константа, не зависящая от $и(t)$ $u v(t)$. Пусть $\mathscr{L}=\left\{l_{j}: j=1, \ldots, N\right\}$ - некоторая совокупность линейных непрерывных функиионалов на $V$, обладающая свойством (1.15) с ве $<\sqrt{\nu / R}$. Тогда $\mathscr{L}$ является множсеством $(V, \bar{W})$-определяющих функчионалов задачи (1.1). 
ДокАЗАТЕЛЬСтво. Так же, как и при доказательстве следствия 1.1 , используя (1.16), получаем (1.17) для всех $t \geqslant s$. Условие (1.26) позволяет убедиться в том, что для функции $\psi(t)=\nu(1+\delta)^{-1} \varepsilon_{\mathscr{L}}^{-2}-\phi(t)$ вьполнены соотношения (1.22) и (1.23) при условии, что $\varepsilon \mathscr{L}<\sqrt{\nu / R}$, а $\delta>0$ - достаточно мало. Поэтому теорема 1.2 дает искомое утверждение.

Применение следствий 1.1 и 1.2 для доказательства конечности множества $\mathscr{L}$ определяюших элементов требует вьполнения неравенств $(1.13),(1.14)$ или $(1.25),(1.26)$, а также соотношения (1.15) с достаточно малой константой $\varepsilon \mathscr{L}$. Как показывает анализ конкретных примеров (см. [6]-[21]), вьполнение оценок $(1.13),(1.14),(1.25),(1.26)$ связано, в основном, со свойствами диссипативности системы. Методы их получения достаточно хорошо разработаны (см. [1]-[3]), соответствуюшие константы $\nu$ и $R$ во многих случаях либо близки к оптимальным, либо явно оцениваются через параметры, входяшие в уравнения. Поэтому вопрос об описании конечных наборов функционалов, однозначно определяющих асимптотическую динамику процесса, сводится к изучению множеств функционалов, для которых оценка (1.15) справедлива при достаточно малых $\varepsilon \mathscr{L}$. Это изучение удобно провести, опираясь на понятие дефекта полноты системы функционалов относительно пары пространств (см. $\S 2$ данной статьи).

Другой класс утверждений о существовании определяющих элементов получается для задач, обладаюших асимптотически полными инерциальными многообразиями (ИМ) (о понятии и свойствах инерциальных многообразий см., например, [2], [4], [23]-[26] и ссылки, содержашиеся в этих работах). Приведенное ниже определение ИМ несколько отличается от стандартного. Это сделано для того, чтобы охватить максимально широкий класс уравнений.

ОПрЕДЕЛЕНИЕ 1.3. Пусть $\mathscr{M}_{t}$ - конечномерная поверхность в пространстве $V \times \mathbb{R}$ вида

$$
\mathscr{M}_{t}=\{p+\Phi(p, t): p \in P V, \Phi: P V \times \mathbb{R} \rightarrow(I-P) V\},
$$

где $P$ - конечномерный проектор в $V$, а $\Phi(\cdot, \cdot)$ - непрерывное отображение, удовлетворяющее условию Лигшица

$$
\left\|\Phi\left(p_{1}, t\right)-\Phi\left(p_{2}, t\right)\right\|_{V} \leqslant L \cdot\left\|p_{1}-p_{2}\right\|_{V}
$$

для всех $p_{j} \in P V$ и $t \in \mathbb{R}$, где $L$ - положительная константа. Пусть $\mathscr{W}$ - некоторьй класс решений задачи (1.1), определенных на полуоси $\mathbb{R}_{+}$и обладающих тем свойством, что для каждого $u(t) \in \mathscr{W}$ существует момент $t_{0}$ такой, что

$$
u(t) \in C\left(t_{0},+\infty ; V\right) .
$$

Тогда $\mathscr{M}_{t}$ называется асимптотически полным инерциальным многообразием задачи (1.1) в классе решений $\mathscr{W}$, если

а) поверхность $\mathscr{M}_{t}$ является инвариантной, т.е. для любого $u \in \mathscr{W}$ из условия $u(s) \in \mathscr{M}_{s}$ вытекает, что $u(t) \in \mathscr{M}_{t}$ для всех $t \geqslant s ;$

б) вьполнено условие асимптотической полноты, т.е. для любого $u \in \mathscr{W}$ сушествует решение $u^{*} \in \mathscr{W}$ такое, что $u^{*}(t) \in \mathscr{M}_{t}$ для всех $t>0$ и

$$
\left\|u(t)-u^{*}(t)\right\|_{V} \leqslant C \cdot e^{-\gamma t}
$$

при $t \geqslant t_{0}$, где $C, \gamma$ и $t_{0}$ - некоторые положительные константы. 
ТеОРема 1.3. Пусть $V$ и $V$ непрерывно вложсено в $H$, и пусть $\mathscr{W}$ - класс решений задачи (1.1), обладающих свойством (1.29). Предположим, что задача (1.1) в классе $\mathscr{W}$ имеет асимптотически полное инерииальное многообразие $\mathscr{M}_{t}$, причем проектор $P$, фигурирующий в определении $\mathscr{M}_{t}$, продолэсается по непрерывности до отображения из $H$ в $V$, т.е. существует константа $\Lambda=\Lambda(P)>0$ такая, что

$$
\|P v\|_{V} \leqslant \Lambda \cdot\|v\|_{H}, \quad v \in V .
$$

Тогда если $\mathscr{L}=\left\{l_{j}: j=1, \ldots, N\right\}$ - множество линейных непрерывных функиионалов на $V$, обладающее свойством (1.15) с константой $\varepsilon \mathscr{L}$, удовлетворяющей условию

$$
\varepsilon \mathscr{L}<(1+L)^{-1} \Lambda^{-1}
$$

то отображсение $r_{\mathscr{L}}$, действующее из $V$ в $\mathbb{R}^{N}$ по формуле $r_{\mathscr{L}} u=\left\{l_{j}(u)\right\}_{j=1}^{N}$, является липшицевым гомеоморфизмом из $\mathscr{M}_{t}$ в $\mathbb{R}^{N}$ при каждом $t \in \mathbb{R}$. При этом мноэсество функиионалов $\mathscr{L}$ для задачи (1.1) является асимптотически определяющим в следующем смысле: для любой пары решений $u_{1}, u_{2} \in \mathscr{W}$ из условия

$$
\lim _{t \rightarrow \infty}\left|l_{j}\left(u_{1}(t)\right)-l_{j}\left(u_{2}(t)\right)\right|=0 \quad \text { для } \quad j=1, \ldots, N
$$

вытекает, что

$$
\lim _{t \rightarrow \infty}\left\|u_{1}(t)-u_{2}(t)\right\|_{V}=0
$$

Отметим, что утверждение, подобное теореме 1.3, было установлено ранее для уравнения Курамото-Сивашинского

$$
u_{t}+u_{x x x x}+u_{x x}+u \cdot u_{x}=0, \quad x \in(0, L), \quad t>0
$$

с периодическими на $[0, L]$ граничными условиями в работе [12] в том случае, когда $\left\{l_{j}\right\}$ - множество равномерно распределенных на $[0, L]$ узлов, т.е. $l_{j}(u)=u(j h)$, где $h=L / N, j=1, \ldots, N$. Некоторое обсуждение общей ситуации имеется также в [17].

ДокАЗАТЕЛЬСТво ТЕОРемЫ 1.3. Пусть $u_{1}, u_{2} \in \mathscr{M}_{t}$. Тогда

$$
u_{j}=P u_{j}+\Phi\left(P u_{j}, t\right), \quad j=1,2 .
$$

При этом (1.28) дает, что

$$
\left\|u_{1}-u_{2}\right\|_{V} \leqslant(1+L) \cdot\left\|P u_{1}-P u_{2}\right\|_{V}, \quad u_{1}, u_{2} \in \mathscr{M}_{t} .
$$

Следовательно, используя (1.15) и (1.31), получаем, что

$$
\left\|u_{1}-u_{2}\right\|_{H} \leqslant C_{\mathscr{L}} \cdot \max _{j=1, \ldots, N}\left|l_{j}\left(u_{1}-u_{2}\right)\right|+\varepsilon \mathscr{L} \cdot(1+L) \cdot \Lambda \cdot\left\|u_{1}-u_{2}\right\|_{H} \cdot
$$


Отсюда при условии (1.32) получаем, что

$$
\left\|u_{1}-u_{2}\right\|_{H} \leqslant C_{\mathscr{L}} \cdot(1-\varepsilon \mathscr{L} \cdot(1+L) \cdot \Lambda)^{-1} \cdot \max _{j=1, \ldots, N}\left|l_{j}\left(u_{1}-u_{2}\right)\right| .
$$

С другой стороны, непрерьвность функционалов $l_{j}$ и неравенства $(1.35),(1.31)$ дают, что

$$
\left|l_{j}\left(u_{1}-u_{2}\right)\right| \leqslant \widetilde{C}_{\mathscr{L}} \cdot(1+L) \cdot \Lambda \cdot\left\|u_{1}-u_{2}\right\|_{H} \cdot
$$

Из оценок (1.36) и (1.37) вытекает, что

$$
c_{1, \mathscr{L}} \cdot\left\|u_{1}-u_{2}\right\|_{H} \leqslant \max _{j=1, \ldots, N}\left|l_{j}\left(u_{1}-u_{2}\right)\right| \leqslant c_{2, \mathscr{L}} \cdot\left\|u_{1}-u_{2}\right\|_{H} .
$$

Кроме того, (1.31) и (1.35) дают, что

$$
c_{3} \cdot\left\|u_{1}-u_{2}\right\|_{V} \leqslant\left\|u_{1}-u_{2}\right\|_{H} \leqslant c_{4} \cdot\left\|u_{1}-u_{2}\right\|_{V}, \quad u_{1}, u_{2} \in \mathscr{M}_{t} .
$$

Поэтому соотношение (1.38) означает, что отображение $r_{\mathscr{L}}$ является липшицевьг гомеоморфизмом из $\mathscr{M}_{t}$ в $\mathbb{R}^{N}$ при каждом $t \in \mathbb{R}$.

Докажем теперь второе утверждение теоремы 1.3. Пусть $u_{1}(t), u_{2}(t) \in \mathscr{W}$. С помошью (1.35) легко убедиться в том, что

$$
\left\|u_{1}(t)-u_{2}(t)\right\|_{V} \leqslant(1+L) \cdot\left\|P u_{1}(t)-P u_{2}(t)\right\|_{V}+\sum_{j=1,2}\left\|(1-P) u_{j}(t)-\Phi\left(P u_{j}(t), t\right)\right\|_{V} .
$$

Из свойства асимптотической полноты (1.30) вытекает, что

$$
\left\|(1-P) u_{j}(t)-\Phi\left(P u_{j}(t), t\right)\right\|_{V} \leqslant C \cdot e^{-\gamma t}, \quad t>t_{0} .
$$

Поэтому (1.31) приводит к оценке

$$
\left\|u_{1}(t)-u_{2}(t)\right\|_{V} \leqslant(1+L) \cdot \Lambda\left\|u_{1}(t)-u_{2}(t)\right\|_{H}+C \cdot e^{-\gamma t}, \quad t>t_{0} .
$$

Следовательно, при условии (1.32) соотношение (1.15) дает, что

$$
\left\|u_{1}(t)-u_{2}(t)\right\|_{V} \leqslant A_{\mathscr{L}} \cdot \max _{j=1, \ldots, N}\left|l_{j}\left(u_{1}(t)-u_{2}(t)\right)\right|+B \mathscr{L} \cdot e^{-\gamma t}, \quad t>t_{0},
$$

где $A_{\mathscr{L}}$ и $B \mathscr{L}$ - положительные числа. Значит, условие (1.33) влечет (1.34). Теорема 1.3 доказана.

Отметим, что, так же, как и при доказательстве следствий 1.1 и 1.2, соотношение $(1.15)$ с достаточно малой константой $\varepsilon \mathscr{L}$ играет важную роль в теореме 1.3 при описании функционалов, однозначно определяющих динамику системы. Анализу ситуаций, для которых оценка (1.15) возможна при достаточно малых $\varepsilon \mathscr{L}$, посвяшены следуюшие два параграфа. Представленные там подходы позволяют, в частности, получать оценки снизу числа функционалов, для которых справедливо неравенство вида (1.15) при фиксированном $\varepsilon \mathscr{L}>0$. 


\section{§ 2. Дефект полноты множества функционалов}

Для характеризации множеств определяюших элементов будем использовать следуюшее понятие дефекта полноты.

ОПРЕДЕЛЕНИЕ 2.1. Пусть $V$ и $H$ - рефлексивные банаховы пространства, $V$ непрерьвно и плотно вложено в $H$. Дефектом полноты множества $\mathscr{L}$ линейных функшионалов на $V$ относительно $H$ назьвается величина

$$
\varepsilon_{\mathscr{L}}(V, H)=\sup \left\{\|w\|_{H}: w \in V, l(w)=0, l \in \mathscr{L},\|w\|_{V} \leqslant 1\right\} .
$$

Отметим, что конечномерность линейной оболочки $\operatorname{Lin} \mathscr{L}$ множества $\mathscr{L}$ пока не предполагается. Очевидно также, что $\varepsilon \mathscr{L}_{1}(V, H) \geqslant \varepsilon \mathscr{L}_{2}(V, H)$ при условии Lin $\mathscr{L}_{1} \subset$ $\operatorname{Lin} \mathscr{L}_{2}$. Кроме того, $\varepsilon \mathscr{L}(V, H)=0$ тогда и только тогда, когда система функщионалов $\mathscr{L}$ полна на $V$, т.е. из условия $l(w)=0$ для всех $l \in \mathscr{L}$ вытекает, что $w=0$.

Следующее утверждение устанавливает связь дефекта полноты и оценки (1.15), играющей важную роль в теории определяющих функционалов. Оно означает, что предположение малости параметра $\varepsilon \mathscr{L}$ в условиях следствий $1.1,1.2$ и теоремы 1.3 можно заменить соответствуюшим требованием относительно дефекта полноты $\varepsilon \mathscr{L}(V, H)$.

ТеОРема 2.1. Пусть $\varepsilon \mathscr{L}=\varepsilon \mathscr{L}(V, H)$ дефект полноты множества $\mathscr{L}$ линейных функиионалов на $V$ относительно $H$. Тогда существует положительная константа $C_{\mathscr{L}}$ такая, что

$$
\|w\|_{H} \leqslant C_{\mathscr{L}} \cdot \sup \left\{|l(w)|: l \in \overline{\mathscr{L}},\|l\|_{*} \leqslant 1\right\}+\varepsilon \mathscr{L} \cdot\|w\|_{V}
$$

для любого $w \in V$, где $\overline{\mathscr{L}}$ - замкнутая линейная оболочка множества $\mathscr{L}$ в $V^{*}$, $\|\cdot\|_{*}-$ норма в $V^{*}$.

ДокАЗАТЕльСТво. Из определения (2.1) вытекает, что

$$
\|w\|_{H} \leqslant \varepsilon \mathscr{L} \cdot\|w\|_{V} \text { для всех } w \in \mathscr{L}^{\perp},
$$

где $\mathscr{L}^{\perp} \equiv\{v \in V: l(v)=0, l \in \mathscr{L}\}$ - аннулятор для $\mathscr{L}$. Так как $l(w)=0$ для всех $w \in \mathscr{L}^{\perp}$ и $l \in \widehat{\mathscr{L}}$, то (2.3) означает, что соотношение $(2.2)$ справедливо для $w \in \mathscr{L}^{\perp}$. Пусть теперь $w \notin \mathscr{L}^{\perp}$. Тогда сушествует элемент $u \in \mathscr{L}^{\perp}$ такой, что $\|w-u\|=$ $\operatorname{dist}_{V}\left(w, \mathscr{L}^{\perp}\right)$. Из непрерьвности вложения $V$ в $H$ и из (2.3) вытекает, что

$$
\|w\|_{H} \leqslant\|u\|_{H}+\|w-u\|_{H} \leqslant \varepsilon \mathscr{L} \cdot\|u\|_{V}+C \cdot\|w-u\|_{V} .
$$

Поэтому неравенство $\|u\|_{V} \leqslant\|w\|_{V}+\|w-u\|_{V}$ дает, что

$$
\|w\|_{H} \leqslant \varepsilon \mathscr{L} \cdot\|w\|_{V}+C_{\mathscr{L}} \cdot\|w-u\|_{V}
$$

с некоторой константой $C_{\mathscr{L}}>0$. Далее, с помощью теоремы Хана-Банаха легко построить (см., например, [27]) на пространстве $V$ непрерьвньй линейный функционал $l_{0}$, обладающий свойствами:

$$
l_{0}(w-u)=\|w-u\|_{V}, \quad\left\|l_{0}\right\|_{*}=1, \quad l_{0}(v)=0 \text { для всех } v \in \mathscr{L}^{\perp} .
$$

Очевидно, что $l_{0} \in \overline{\mathscr{L}}$. При этом из $(2.4)$ получаем, что

$$
\|w\|_{H} \leqslant \varepsilon \mathscr{L} \cdot\|w\|_{V}+C_{\mathscr{L}} \cdot l_{0}(w)
$$

Поэтому имеет место соотношение (2.2). Теорема 2.1 доказана.

Следуюшее утверждение дает характеризацию дефекта полноты в спектральных терминах для случая, когда $V$ и $H$ - гильбертовы пространства. 
ТеОРема 2.2. Пусть $V$ и $H$ - сепарабельные гильбертовы пространства, $V$ компактно и плотно вложсено в $H$. Пусть $K$ - самосопряженный положительный вполне непрерывный оператор в пространстве $V$, определяемый равенством $(K u, v)_{V}=(u, v)_{H}$ для $u, v \in V$. Тогда дефект полноты $\varepsilon \mathscr{L}(V, H)$ множсества $\mathscr{L}$ линейных функиионалов на $V$ может быть вычислен по формуne

$$
\varepsilon_{\mathscr{L}}(V, H)=\sqrt{\mu_{\max }\left(P_{\mathscr{L}} K P_{\mathscr{L}}\right)}
$$

где $P_{\mathscr{L}}$ - оператор ортогонального проектирования в пространстве $V$ на аннулятор $\mathscr{L}^{\perp}=\{v \in V: l(v)=0, l \in \mathscr{L}\}, \mu_{\max }(S)$ - максимальное собственное число оператора $S$.

ДоКАЗАТЕЛЬСтво. По определению 2.1 имеем, что

$$
\varepsilon \mathscr{L}(V, H)=\sup \left\{\|w\|_{H}: w \in B \mathscr{L}\right\}
$$

где $B_{\mathscr{L}}=\mathscr{L}^{\perp} \cap\left\{v:\|v\|_{V} \leqslant 1\right\}-$ единичньй шар в $\mathscr{L}^{\perp}$. Очевидно, что $B_{\mathscr{L}}-$ компакт в $H$. Поэтому сушествует элемент $u_{0} \in B \mathscr{L}$ такой, что $\left\|u_{0}\right\|_{V}=1$ и

$$
\varepsilon_{\mathscr{L}}(V, H)^{2}=\left\|u_{0}\right\|_{H}^{2}=\left(K u_{0}, u_{0}\right)_{V}
$$

Этот Элемент доставляет максимум функции $(K u, u)_{V}$ на множестве $B \mathscr{L}$, и следовательно, для любого $v \in \mathscr{L}^{\perp}$ и любого $s \in \mathbb{R}$ справедливо соотношение

$$
\frac{\left(K\left(u_{0}+s v\right), u_{0}+s v\right)_{V}}{\left\|u_{0}+s v\right\|_{V}^{2}} \leqslant \mu \equiv\left(K u_{0}, u_{0}\right)_{V} .
$$

Поэтому

$$
\left(K\left(u_{0}+s v\right), u_{0}+s v\right)_{V}-\mu\left\|u_{0}+s v\right\|_{V}^{2} \leqslant 0 .
$$

Следовательно,

$$
s^{2}\left\{(K v, v)_{V}-\mu\|v\|_{V}^{2}\right\}+2 s\left\{\left(K u_{0}, v\right)_{V}-\mu\left(u_{0}, v\right)_{V}\right\} \leqslant 0
$$

для всех $v \in \mathscr{L}^{\perp}$ и $s \in \mathbb{R}$. Отсюда легко получить, что

$$
\left(K u_{0}, v\right)_{V}-\mu\left(u_{0}, v\right)_{V}=0 \text { для всех } v \in \mathscr{L}^{\perp}
$$

Поэтому $P_{\mathscr{L}} K P_{\mathscr{L}} u_{0}=\mu u_{0}$, т.е. $\mu=\left(K u_{0}, u_{0}\right)_{V}=\left\|u_{0}\right\|_{H}^{2}$ является собственным числом оператора $P_{\mathscr{L}} K P_{\mathscr{L}}$. Ясно, что это максимальное собственное число. Таким образом, теорема 2.2 доказана.

Ситуация, описанная в следуюшем утверждении, часто возникает в приложениях в контексте так называемых определяюших мод (см. [6]-[8] и др.). 
СлЕДСТВИЕ 2.1. Пусть выполнены условия теоремы 2.2. Обозначим через $\left\{e_{i}\right\}$ ортонормированный базис в пространстве $V$, состоящий из собственных векторов оператора $K$ :

$$
K e_{i}=\mu_{i} e_{i}, \quad\left(e_{i}, e_{j}\right)_{V}=\delta_{i j}, \quad \mu_{1} \geqslant \mu_{2} \geqslant \ldots, \quad \lim _{N \rightarrow \infty} \mu_{N}=0 .
$$

Тогда дефект полноты системы функиионалов

$$
\mathscr{L}=\left\{l_{j} \in V^{*}: l_{j}(v)=\left(v, e_{j}\right)_{V}, j=1,2, \ldots, N\right\}
$$

может бить виччслен по формуле $\varepsilon \mathscr{L}(V, H)=\sqrt{\mu_{N+1}}$.

Для доказательства этого утверждения достаточно заметить, что в данном случае $P_{\mathscr{L}}$ - ортопроектор на замкнутую линейную оболочку элементов $\left\{e_{j}: j \geqslant N+1\right\}$ и что $P_{\mathscr{L}}$ коммутирует с $K$.

Следующее утверждение устанавливает связь дефекта полноты с некоторьми понятиями общей теории аппроксимаций (см., например, [28], [29] и приведенные в этих работах ссылки). Оно будет в дальнейшем использоваться для получения оценок сверху величины $\varepsilon \mathscr{L}$.

ТеОрема 2.3. Пусть выполнены условия теоремы 2.2. Предположим, что $\mathscr{L}$ - некоторое множество линейных функционалов на $V$ и $\mathscr{R}_{\mathscr{L}}$ - семейство линейных ограниченных операторов $R$, отображсающи $V$ в $H$ и таких, что $R v=0$ для всех $v \in \mathscr{L}^{\perp}$. Пусть $e_{V}^{H}(R)$ - глобальная аппроксимационная ошибка в $H$, возникающая при приближении әлементов $v \in V$ әлементами $R v$ и определяемая равенством:

$$
e_{V}^{H}(R)=\sup \left\{\|u-R u\|_{H}:\|u\|_{V} \leqslant 1\right\} .
$$

Тогда

$$
\varepsilon_{\mathscr{L}}(V, H)=\min \left\{e_{V}^{H}(R): R \in \mathscr{R}_{\mathscr{L}}\right\}
$$

ДокАЗАТЕльСТво. Пусть $R \in \mathscr{R} \mathscr{L}$. Тогда очевидно, что

$$
\|u-R u\|_{H} \leqslant e_{V}^{H}(R) \cdot\|u\|_{V} \text { для всех } u \in V .
$$

Поэтому для $u \in \mathscr{L}^{\perp}$ имеем, что $\|u\|_{H} \leqslant e_{V}^{H}(R) \cdot\|u\|_{V}$. Отсюда вытекает, что $\varepsilon \mathscr{L}(V, H) \leqslant e_{V}^{H}(R)$ для всех $R \in \mathscr{R} \mathscr{L}$. Покажем теперь, что существует оператор $R_{0} \in \mathscr{R}_{\mathscr{L}}$ такой, что $\varepsilon \mathscr{L}(V, H)=e_{V}^{H}\left(R_{0}\right)$. Из $(2.5)$ вытекает, что

$$
\varepsilon \mathscr{L}(V, H)=\left\|K^{1 / 2} P_{\mathscr{L}}\right\|_{L(V, V)} \equiv \sup \left\{\left\|K^{1 / 2} P_{\mathscr{L}} u\right\|_{V}:\|u\|_{V} \leqslant 1\right\},
$$

где $P_{\mathscr{L}}$ - оператор ортогонального проектирования в пространстве $V$ на аннулятор $\mathscr{L}^{\perp},\|\cdot\|_{L(V, V)}-$ норма в пространстве линейных ограниченных операторов, действующих в $V$. Поэтому из определения оператора $K$ (см. формулировку теоремы 2.1 ) вытекает, что

$$
\varepsilon \mathscr{L}(V, H)=\sup \left\{\left\|P_{\mathscr{L}} u\right\|_{H}:\|u\|_{V} \leqslant 1\right\}=e_{V}^{H}\left(I-P_{\mathscr{L}}\right) .
$$

Ясно, что $I-P_{\mathscr{L}} \in \mathscr{R}_{\mathscr{L}}$. Поэтому можно положить $R_{0}=I-P_{\mathscr{L}}$. Теорема 2.3 доказана. 
ЗАмечание 2.1. Пусть $\mathscr{L}=\left\{l_{j}: j=1,2, \ldots, N\right\}$ - конечньй набор линейно независимых функционалов на $V$. Тогда в условиях теорем 2.2 и 2.3 существует набор элементов $\left\{\phi_{k}: k=1,2, \ldots, N\right\}$ из $V$ такой, что $l_{j}\left(\phi_{k}\right)=\delta_{j k}$, где $\delta_{j k}=0$, если $k \neq j$, и $\delta_{j k}=1$ при $k=j$, а оператор $R_{0}$, фигурируюший в доказательстве теоремы 2.3 , имеет вид $R_{0} u=\sum_{j=1}^{N} l_{j}(u) \phi_{j}$. Соотношение (2.10) при этом дает, что

$$
\left\|u-R_{0} u\right\|_{H} \leqslant \varepsilon \mathscr{L}(V, H) \cdot\|u\|_{V} .
$$

Таким образом, на пространстве $V$ сушествует лагранжев $\left(l_{j}\left(\phi_{k}\right)=\delta_{j k}\right)$ интерполяционньй оператор $R_{0}$ с погрешностью интерполящии, определяемой дефектом полноты $\varepsilon \mathscr{L}(V, H)$.

Для получения оценок снизу дефекта полноты важную роль играет понятие поперечника вложения (см., например, [28], [29], а также монографии [30] и [31], в которых приведены результаты вычисления поперечников для ряда функциональных пространств). Дадим следуюшее определение.

ОПРЕДЕЛЕНИЕ 2.2. Пусть $V$ и $H$ - сепарабельные гильбертовы пространства, $V$ непрерьвно и плотно вложено в $H$. Тогда $N$-поперечником Колмогорова вложения $V$ в $H$ назьвается величина

$$
\kappa_{N}=\kappa_{N}(V, H)=\inf \left\{e_{V}^{H}(F): F \in \mathscr{F}_{N}\right\},
$$

где $\mathscr{F}_{N}$ - совокупность всех $N$-мерных подпространств $F$ пространства $V$, а $e_{V}^{H}(F)-$ глобальная ошибка аппроксимации в $H$ элементов из $V$ элементами подпространства $F$, определяемая равенством:

$$
e_{V}^{H}(F)=\sup \left\{\operatorname{dist}_{H}(v, F):\|v\|_{V} \leqslant 1\right\} .
$$

Другими словами, $N$-поперечник Колмогорова $\kappa_{N}$ вложения $V$ в $H$ совпадает с минимально возможной глобальной ошибкой аппоксимации в $H$ элементов из $V$ элементами $N$-мерного подпространства.

ТеОРема 2.4. Пусть $V$ и $H$ - сепарабельнье гильбертовьи пространства, причем $V$ плотно и компактно вложено в $H$. Тогда

$$
\kappa_{N}(V, H)=\min \left\{\varepsilon_{\mathscr{L}}(V, H): \mathscr{L} \subset V^{*}, \operatorname{dim} \operatorname{Lin} \mathscr{L}=N\right\}=\sqrt{\mu_{N+1}},
$$

әде $\left\{\mu_{j}\right\}$ - упорядоченные по невозрастанию собственные числа оператора $K$, определяемого равенством $(K u, v)_{V}=(u, v)_{H}$ для $u, v \in V$.

Эта теорема, дополненная равенством (2.9), по существу представляет собой некоторую удобную для дальнейшего переформулировку известных свойств $s$-чисел вполне непрерьвных операторов (см. [32, гл. $2, \S 2])$. Однако, ради замкнутости изложения здесь приведено доказательство, не зависимое от [32]. 
ДокАЗАТЕЛЬСТво. Покажем сначала, что

$$
\min \left\{\varepsilon_{\mathscr{L}}(V, H): \mathscr{L} \subset V^{*} ; \operatorname{dim} \operatorname{Lin} \mathscr{L}=N\right\}=\sqrt{\mu_{N+1}} .
$$

Ввиду следствия 2.1 достаточно убедиться в том, что $\varepsilon \mathscr{L}(V, H) \geqslant \sqrt{\mu_{N+1}}$ для всех $\mathscr{L}=\left\{l_{j}: j=1,2, \ldots, N\right\}$, где $l_{j}$ - линейно независимые функционалы на $V$. Из определения (2.1) вытекает, что

$$
\left[\varepsilon_{\mathscr{L}}(V, H)\right]^{2} \geqslant\|u\|_{H}^{2}=\sum_{j=1}^{\infty} \mu_{j}\left(u, e_{j}\right)_{V}^{2}
$$

для любых $u \in V$ таких, что $\|u\|_{V}=1$ и $l_{j}(u)=0, j=1,2, \ldots, N$. Здесь $\left\{e_{j}\right\}-$ ортонормированный базис в пространстве $V$, обладаюший свойствами (2.6). Возьмем в (2.14) в качестве элемента $u$ вектор $u=\sum_{j=1}^{N+1} c_{j} e_{j}$, где числа $c_{k}$ подобраны так, что $\|u\|_{V}=1$ и $l_{j}(u)=0, j=1,2, \ldots, N$. Тогда из (2.14) имеем, что

$$
\left[\varepsilon_{\mathscr{L}}(V, H)\right]^{2} \geqslant \sum_{j=1}^{N+1} \mu_{j} c_{j}^{2} \geqslant \mu_{N+1} \sum_{j=1}^{N+1} c_{j}^{2}=\mu_{N+1}\|u\|_{V}^{2}=\mu_{N+1} .
$$

Таким образом, соотношение (2.13) доказано.

Проверим теперь, что $\kappa_{N}(V, H)=\min \{\varepsilon \mathscr{L}\}$. Предположим, что $\mathscr{L} \subset V^{*}$ и $\operatorname{dim} \operatorname{Lin} \mathscr{L}=N$. Пусть, как и ранее, $P_{\mathscr{L}}-$ оператор ортогонального проектирования в пространстве $V$ на аннулятор $\mathscr{L}^{\perp}=\{v \in V: l(v)=0, l \in \mathscr{L}\}$, и пусть $Q_{\mathscr{L}}=I-P \mathscr{L}$. Очевидно, что $Q_{\mathscr{L}} V$ изоморфно $\operatorname{Lin} \mathscr{L}$. Поэтому $\operatorname{dim} Q_{\mathscr{L}} V=N$. Следовательно, (2.10) дает, что

$$
\varepsilon_{\mathscr{L}}(V, H)=e_{V}^{H}\left(Q_{\mathscr{L}}\right) \geqslant \sup \left\{\operatorname{dist}_{H}\left(u, Q_{\mathscr{L}} V\right):\|u\|_{V} \leqslant 1\right\} \geqslant \kappa_{N}
$$

для всех $\mathscr{L} \subset V^{*}$ таких, что $\operatorname{dim} \operatorname{Lin} \mathscr{L}=N$. Наоборот, пусть $F$ - некоторое $N$-мерное подпространство в $V$, и пусть $\left\{f_{j}: j=1, \ldots, N\right\}$ - его базис, ортонормированный в пространстве $H$. Положим

$$
\mathscr{L}_{F}=\left\{l_{j} \in V^{*}: l_{j}(v)=\left(v, f_{j}\right)_{H}, j=1,2, \ldots, N\right\} .
$$

Пусть $Q_{H, F}$ - оператор ортогонального проектирования в пространстве $H$ на $F$. Очевидно, что

$$
Q_{H, F} u=\sum_{j=1}^{N}\left(u, f_{j}\right)_{H} f_{j} .
$$

Поэтому если $u \in \mathscr{L}_{F}^{\perp}$, то $Q_{H, F} u=0$. Кроме того,

$$
\left\|Q_{H, F} u\right\|_{V} \leqslant \sum_{j=1}^{N}\left|\left(u, f_{j}\right)_{H}\right| \cdot\left\|f_{j}\right\|_{V} \leqslant\left\{\sum_{j=1}^{N}\left\|f_{j}\right\|_{H} \cdot\left\|f_{j}\right\|_{V}\right\} \cdot\|u\|_{H} .
$$

Отсюда вытекает, что $Q_{H, F}$ - ограниченный оператор в $V$. Поэтому, применяя теорему 2.3 , находим, что

$$
\varepsilon \mathscr{L}_{F}(V, H) \leqslant e_{V}^{H}\left(Q_{H, F}\right)=\sup \left\{\left\|u-Q_{H, F} u\right\|_{H}:\|u\|_{V} \leqslant 1\right\} .
$$


Но $\left\|u-Q_{H, F} u\right\|_{H}=\operatorname{dist}_{H}(u, F)$. Следовательно,

$$
\min \left\{\varepsilon_{\mathscr{L}}\right\} \leqslant \varepsilon_{\mathscr{L}_{F}}(V, H) \leqslant e_{V}^{H}(F)
$$

для любого $N$-мерного подпространства $F$ в $V$. Из $(2.15)$ и (2.16) вытекает, что

$$
\kappa_{N}(V, H)=\min \left\{\varepsilon_{\mathscr{L}}(V, H): \mathscr{L} \subset V^{*} ; \operatorname{dim} \operatorname{Lin} \mathscr{L}=N\right\}
$$

Вместе с (2.13) это соотношение завершает доказательство теоремы 2.4.

В $\S 3$ нам понадобится следуюшее свойство дефекта полноты.

Лемма 2.1. Пусть $V, W$ и $H$-рефлексивнье банаховь пространства такие, что $V \subset W \subset H$, причем все вложения непрерывны и плотны. Предположим, что с некоторыми константами $a_{\theta}>0 u 0<\theta<1$ справедливо неравенство

$$
\|u\|_{W} \leqslant a_{\theta}\|u\|_{H}^{\theta}\|u\|_{V}^{1-\theta}, \quad u \in V .
$$

Тогда для любого множества $\mathscr{L}$ линейных функционалов на $W$ имеет место оценка

$$
\left[a_{\theta}^{-1} \varepsilon_{\mathscr{L}}(V, W)\right]^{\frac{1}{\theta}} \leqslant \varepsilon \mathscr{L}(V, H) \leqslant\left[a_{\theta} \varepsilon \mathscr{L}(W, H)\right]^{\frac{1}{1-\theta}} .
$$

ДокАЗАТЕЛЬСТвО. Пусть $u \in \mathscr{L}_{W}^{\perp}=\{w \in W: l(w)=0\}$. Тогда из определения 2.1 и неравенства $(2.17)$ вытекает, что

$$
\|u\|_{H} \leqslant \varepsilon \mathscr{L}(W, H)\|u\|_{W} \leqslant a_{\theta} \varepsilon \mathscr{L}(W, H)\|u\|_{H}^{\theta}\|u\|_{V}^{1-\theta}
$$

для всех $u \in \mathscr{L}_{V}^{\stackrel{\perp}{ }}=\mathscr{L}_{W}^{\perp} \cap V$. Поэтому

$$
\|u\|_{H} \leqslant\left[a_{\theta} \varepsilon \mathscr{L}(W, H)\right]^{\frac{1}{1-\theta}}\|u\|_{V}, \quad u \in \mathscr{L}_{V}^{\perp} .
$$

Точно так же имеем, что

$$
\|u\|_{W} \leqslant a_{\theta}\|u\|_{H}^{\theta}\|u\|_{V}^{1-\theta} \leqslant a_{\theta}[\varepsilon \mathscr{L}(V, H)]^{\theta}\|u\|_{V}, \quad u \in \mathscr{L}_{V}^{\perp} .
$$

Из двух последних неравенств нетрудно извлечь оценку (2.18). 


\section{§. Оценки дефекта полноты в соболевских пространствах}

В этом параграфе приведено несколько важных с точки зрения приложений семейств функщионалов на соболевских пространствах и даны оценки соответствующих деффектов полноты.

Предположим, что $\Omega$ - ограниченная область в $\mathbb{R}^{\nu}, \nu \geqslant 1, H^{s}(\Omega)$ - соболевское пространство порядка $s$ на $\Omega$ и $H_{0}^{s}(\Omega)$ - замькание в $H^{s}(\Omega)$ множества бесконечно дифференщируемых функщий с компактным носителем в $\Omega$. Напомним, что при цельх неотрицательных значениях $s$ пространства Соболева описываются соотношением:

$$
H^{m}(\Omega)=\left\{f \in L^{2}(\Omega): D^{\alpha} f \in L^{2}(\Omega),|\alpha| \leqslant m\right\},
$$

где $m=0,1,2, \ldots$. Здесь и в дальнейшем используются обозначения:

$$
\begin{gathered}
D^{\alpha} f(x)=\frac{\partial^{|\alpha|} f(x)}{\partial x_{1}^{\alpha_{1}} \partial x_{2}^{\alpha_{2}} \cdots \partial x_{\nu}^{\alpha_{\nu}}} \\
\alpha=\left(\alpha_{1}, \alpha_{2}, \ldots, \alpha_{\nu}\right), \quad \alpha_{k}=0,1,2, \ldots, \quad|\alpha|=\alpha_{1}+\alpha_{2}+\cdots+\alpha_{\nu} .
\end{gathered}
$$

Кроме того, мы полагаем:

$$
\alpha !=\alpha_{1} ! \cdot \alpha_{2} ! \cdot \ldots \cdot \alpha_{\nu} !, \quad x^{\alpha}=x_{1}^{\alpha_{1}} \cdot x_{2}^{\alpha_{2}} \cdot \ldots \cdot x_{\nu}^{\alpha_{\nu}}, \quad|x|=\sqrt{x_{1}^{2}+x_{2}^{2}+\cdots+x_{\nu}^{2}}
$$

для мультииндекса $\alpha=\left(\alpha_{1}, \alpha_{2}, \ldots, \alpha_{\nu}\right)$ и точки $x=\left(x_{1}, \ldots, x_{\nu}\right) \in \mathbb{R}^{\nu}$. Что касается пространств $H^{s}(\Omega)$ для нецелых порядков $s>0$, то они определяются с помошњю интерполяции (см. [31]) между $H^{m_{s}}(\Omega)$ и $H^{m_{s}+1}(\Omega)$, где $m_{s}$ - целая часть $s$. Пусть $\|\cdot\|_{s}$ - норма (интерполящионная при нецельх $s$ ) в $H^{s}(\Omega)$, а $\|\cdot\|$ и $(\cdot, \cdot)$ - норма и скалярное произведение в $L^{2}(\Omega)$.

В дальнейшем используется следуюший вариант интегрального представления Соболева.

Лемма 3.1. Пусть $\Omega$ - область в $\mathbb{R}^{\nu}$, и пусть $\lambda(x)-$ функиия из $L^{\infty}\left(\mathbb{R}^{\nu}\right) c$ компактным носителем supp $\lambda$ такая, что

$$
\operatorname{supp} \lambda \Subset \Omega, \quad \int_{\mathbb{R}^{\nu}} \lambda(x) d x=1 .
$$

Предположим, что область $\Omega$ является звездной относительно носителя $\operatorname{supp} \lambda$, т.е. вместе с кажсдой точкой $x \in \Omega$ области $\Omega$ принадлежст конус

$$
V_{x}=\{z=\tau x+(1-\tau) y: 0 \leqslant \tau \leqslant 1, y \in \operatorname{supp} \lambda\} .
$$

Тогда для любой функции $и(x) \in H^{m}(\Omega)$ справедливо представление

$$
u(x)=P_{m-1}(x, u)+\sum_{|\alpha|=m} \frac{m}{\alpha !} \int_{V_{x}}(x-y)^{\alpha} \cdot K(x, y) \cdot D^{\alpha} u(y) d y,
$$

где $P_{m-1}(x, u)$ - полином переменной $x$ степени $m-1$ вида:

$$
P_{m-1}(x, u)=\sum_{|\alpha| \leqslant m-1} \frac{1}{\alpha !} \int_{\Omega} \lambda(y) \cdot(x-y)^{\alpha} \cdot D^{\alpha} u(y) d y,
$$

а ядро $K(x, y)$ задается формулой

$$
K(x, y)=\int_{0}^{1} \lambda\left(x+\frac{y-x}{s}\right) \frac{d s}{s^{\nu+1}} .
$$


ДокАЗАТЕльСтво. Если формулу Тейлора

$u(x)=\sum_{|\alpha| \leqslant m-1} \frac{(x-y)^{\alpha}}{\alpha !} D^{\alpha} u(y)+m \sum_{|\alpha|=m} \frac{(x-y)^{\alpha}}{\alpha !} \int_{0}^{1} s^{m-1} D^{\alpha} u(x+s \cdot(y-x)) d s$ умножить на $\lambda(y)$ и проинтегрировать по $y$, то после введения новой переменной интегрирования $z=x+s \cdot(y-x)$ получаем утверждение леммы.

Интегральное представление (3.3) позволяет получить следующее обобщение неравенства Пуанкаре.

Лемма 3.2. Пусть для ограниченной области $\Omega$ из $\mathbb{R}^{\nu}$ и функции $\lambda(x)$ из $L^{\infty}\left(\mathbb{R}^{\nu}\right)$ выполнень предположения лем.мы 3.1. Тогда для любой функиии $u(x) \in$ $H^{1}(\Omega)$ справедливо неравенство

$$
\left\|u-\langle u\rangle_{\lambda}\right\| \leqslant \frac{\sigma_{\nu}}{\nu} d^{\nu+1}\|\lambda\|_{L^{\infty}(\Omega)}\|\nabla u\|
$$

где $\langle u\rangle_{\lambda}=\int_{\Omega} \lambda(x) u(x) d x, \sigma_{\nu}-$ площ,адь поверхности единичной сферы в $\mathbb{R}^{\nu}$, $d=\operatorname{diam} \Omega \equiv \sup \{|x-y|: x, y \in \Omega\}$.

ДоКАЗАТЕЛЬСТво. Формула (3.3) при $m=1$ дает, что

$$
u(x)=\langle u\rangle_{\lambda}+\sum_{j=1}^{\nu} \int_{V_{x}}\left(x_{j}-y_{j}\right) \cdot K(x, y) \cdot \frac{\partial u(y)}{\partial y_{j}} d y .
$$

Очевидно, что $\lambda\left(x+\frac{y-x}{s}\right)=0$ при $s^{-1}|x-y| \geqslant d=\operatorname{diam} \Omega$. Поэтому

$$
K(x, y)=\int_{d^{-1}|x-y|}^{1} \lambda\left(x+\frac{y-x}{s}\right) \frac{d s}{s^{\nu+1}} .
$$

Следовательно,

$$
|K(x, y)| \leqslant \delta \cdot|x-y|^{-\nu}, \quad \delta=\delta(\lambda, \nu) \equiv \nu^{-1} d^{\nu}\|\lambda\|_{L^{\infty}(\Omega)} .
$$

Поэтому (3.7) дает, что

$$
\begin{aligned}
\left|u(x)-\langle u\rangle_{\lambda}\right| & \leqslant \delta \int_{\Omega} \frac{|\nabla u(y)|}{|x-y|^{\nu-1}} d y \\
& \leqslant \delta\left(\int_{\Omega} \frac{|\nabla u(y)|^{2}}{|x-y|^{\nu-1}} d y\right)^{1 / 2} \cdot\left(\int_{\Omega} \frac{d y}{|x-y|^{\nu-1}}\right)^{1 / 2} .
\end{aligned}
$$

Пусть $B_{r}(x)=\{y:|x-y| \leqslant r\}$ - шар радиуса $r$ с центром в точке $x$. Тогда очевидно, что

$$
\int_{\Omega} \frac{d y}{|x-y|^{\nu-1}} \leqslant \int_{B_{d}(x)} \frac{d y}{|x-y|^{\nu-1}} \leqslant \sigma_{\nu} d,
$$

где $\sigma_{\nu}$ - площадь поверхности единичной сферы в $\mathbb{R}^{\nu}, d=\operatorname{diam} \Omega$. Поэтому из (3.9) вытекает, что

$$
\left|u(x)-\langle u\rangle_{\lambda}\right|^{2} \leqslant \delta^{2} \sigma_{\nu} d \int_{\Omega} \frac{|\nabla u(y)|^{2}}{|x-y|^{\nu-1}} d y .
$$

После интегрирования по $x$ с помошью (3.10) получаем (3.6). Лемма 3.2 доказана. 
ЛЕмма 3.3. Предположим, что для ограниченной области $\Omega$ из $\mathbb{R}^{\nu}$ и функции $\lambda(x)$ из $L^{\infty}\left(\mathbb{R}^{\nu}\right)$ выполнены условия леммы 3.1. Пусть $m_{\nu}=\left[\frac{\nu}{2}\right]+1$, где [ · ] знак иелой части числа. Тогда для любой функиии и $(x) \in H^{m_{\nu}}(\Omega)$ справедливо неравенство

$$
\max _{x \in \Omega}\left|u(x)-P_{m-1}(x, u)\right| \leqslant c_{\nu} \cdot d^{m_{\nu}+\frac{\nu}{2}}\|\lambda\|_{L^{\infty}(\Omega)} \sum_{|\alpha|=m_{\nu}} \frac{1}{\alpha !} \cdot\left\|D^{\alpha} u\right\|,
$$

әде полином $P_{m-1}(x, u)$ определяется формулой $(3.4), d=\operatorname{diam} \Omega$, а константа $c_{\nu}$ имеет вид $c_{\nu}=\sqrt{\frac{\sigma_{\nu}}{2 m_{\nu}-\nu}} \cdot \frac{m_{\nu}}{\nu}$. Здесь $\sigma_{\nu}-$ площадь поверхности единичной сферы в в $\mathbb{R}^{\nu}$.

ДоКАЗАТЕЛЬСТво. Используя (3.3) и (3.8), находим, что

$$
\begin{aligned}
\left|u(x)-P_{m-1}(x, u)\right| & \leqslant \sum_{|\alpha|=m_{\nu}} \frac{m_{\nu}}{\alpha !} \int_{V_{x}}|x-y|^{m_{\nu}} \cdot|K(x, y)| \cdot\left|D^{\alpha} u(y)\right| d y \\
& \leqslant \sum_{|\alpha|=m_{\nu}} \frac{\delta m_{\nu}}{\alpha !} \int_{\Omega}|x-y|^{m_{\nu}-\nu} \cdot\left|D^{\alpha} u(y)\right| d y \\
& \leqslant \sum_{|\alpha|=m_{\nu}} \frac{\delta m_{\nu}}{\alpha !}\left\|D^{\alpha} u\right\|\left(\int_{\Omega}|x-y|^{2\left(m_{\nu}-\nu\right)} d y\right)^{1 / 2} .
\end{aligned}
$$

Как и ранее, находим, что

$$
\int_{\Omega}|x-y|^{2\left(m_{\nu}-\nu\right)} d y \leqslant \sigma_{\nu} d^{2 m_{\nu}-\nu}\left(2 m_{\nu}-\nu\right)^{-1}
$$

Поэтому имеет место (3.11). Лемма 3.3 доказана.

Лемма 3.4. Предположим, что выполнены условия леммы 3.3. Тогда для любой функции и $(x) \in H^{m_{\nu}}(\Omega)$ и любой точки $x_{*} \in \Omega$ справедливо неравенство

$$
\begin{aligned}
\left\|u-u\left(x_{*}\right)\right\| & \equiv\left(\int_{\Omega}\left|u(x)-u\left(x_{*}\right)\right|^{2} d x\right)^{1 / 2} \\
& \leqslant c_{\nu} \cdot d^{\nu} \cdot\|\lambda\|_{L^{\infty}(\Omega)} \sum_{j=1}^{m_{\nu}} d^{j} \sum_{|\alpha|=j} \frac{1}{\alpha !} \cdot\left\|D^{\alpha} u\right\|,
\end{aligned}
$$

где $c_{\nu}-$ константа, зависящая только от $\nu, d=\operatorname{diam} \Omega$.

ДокаЗАТЕЛЬСтво. Очевидно, что

$$
\left\|u-u\left(x_{*}\right)\right\| \leqslant\left\|u-\langle u\rangle_{\lambda}\right\|+d^{\nu / 2} \cdot\left|\langle u\rangle_{\lambda}-u\left(x_{*}\right)\right|
$$


где, как и выше, $\langle u\rangle_{\lambda}=\int_{\Omega} \lambda(y) u(y) d y$. Структура полинома $P_{m-1}(x, u)$ дает, что

$$
\begin{aligned}
\left|\langle u\rangle_{\lambda}-u(x)\right| & \leqslant\left|u(x)-P_{m_{\nu}-1}(x, u)\right|+\sum_{1 \leqslant|\alpha| \leqslant m_{\nu}-1} \frac{1}{\alpha !} \int_{\Omega}\left|\lambda(y) \cdot(x-y)^{\alpha} \cdot D^{\alpha} u(y)\right| d y \\
& \leqslant\left|u(x)-P_{m_{\nu}-1}(x, u)\right|+\sum_{1 \leqslant|\alpha| \leqslant m_{\nu}-1} \frac{d^{|\alpha|+\nu / 2}}{\alpha !} \cdot\|\lambda\|_{L^{\infty}(\Omega)} \cdot\left\|D^{\alpha} u(y)\right\|
\end{aligned}
$$

для всех $x \in \Omega$. Поэтому оценка (3.12) вытекает из лемм 3.2 и 3.3. Лемма 3.4 доказана.

Доказанные вьше леммы позволяют оценить дефект полноты для двух важных с точки зрения приложений семейств функционалов на соболевских пространствах $H^{s}(\Omega)$ в случае сильно липшицевых областей. Напомним (см., например, [33]), что область $\Omega$ из $\mathbb{R}^{\nu}$ назьвается сильно липшичевой, если для каждого $x \in \partial \Omega$ сушествует окрестность $U$ такая, что в некоторой декартовой системы координат

$$
U \cap \Omega=\left\{x=\left(x_{1}, \ldots, x_{\nu}\right): x_{\nu}<f\left(x_{1}, \ldots, x_{\nu-1}\right)\right\},
$$

где $f(x)$ - функция, удовлетворяюшая условию Липшица. Для сильно липшицевых областей пространство $H^{s}(\Omega)$ состоит из сужений на $\Omega$ функций из $H^{s}\left(\mathbb{R}^{\nu}\right), s>0$ [31], [33].

Следующее утверждение имеет дело с так называемыми обобщенными локальными объемными средними.

ТЕОРема 3.1. Предположим, что ограниченная сильно липшицева область $\Omega$ из $\mathbb{R}^{\nu}$ может быть разбита на подобласти $\left\{\Omega_{j}: j=1,2, \ldots, N\right\}$ так, что

$$
\bar{\Omega}=\bigcup\left\{\bar{\Omega}_{j}: j=1,2, \ldots, N\right\}, \quad \Omega_{j} \bigcap \Omega_{i}=\varnothing, \quad j \neq i,
$$

әде черта означает замыкание множества. Пусть на каждой из подобластей $\Omega_{j}$ задана функция $\lambda_{j}(x)$ из $L^{\infty}\left(\Omega_{j}\right)$ такая, ито

$$
\operatorname{supp} \lambda_{j} \Subset \Omega_{j}, \quad \int_{\Omega_{j}} \lambda_{j}(x) d x=1,
$$

причем $\Omega_{j}$ является звездной относительно носителя $\operatorname{supp} \lambda_{j}$. Определим множество $\mathscr{L}$ обобщенных локальных обгемных средних, отвечающих набору $\mathscr{T}=$ $\left\{\left(\Omega_{j}, \lambda_{j}\right): j=1,2, \ldots, N\right\}$, как семейство функиионалов вида

$$
\mathscr{L}=\left\{l_{j}(u)=\int_{\Omega_{j}} \lambda_{j}(x) u(x) d x, j=1,2, \ldots, N\right\} .
$$

Тогда для $\varepsilon \mathscr{L}(s, \sigma) \equiv \varepsilon_{\mathscr{L}}\left(H^{s}(\Omega), H^{\sigma}(\Omega)\right)$ справедлива оченка

$$
\varepsilon \mathscr{L}(s, \sigma) \leqslant c(\nu, s, \sigma) \cdot \begin{cases}(\Lambda d)^{s-\sigma}, & s>1,0 \leqslant \sigma \leqslant s ; \\ \Lambda^{1-\sigma / s} \cdot d^{s-\sigma}, & 0 \leqslant \sigma \leqslant s \leqslant 1, s \neq 0 .\end{cases}
$$

Здес

$$
\begin{gathered}
\Lambda=\max \left\{d_{j}^{\nu} \cdot\left\|\lambda_{j}\right\|_{L^{\infty}\left(\Omega_{j}\right)}: j=1,2, \ldots, N\right\} ; \\
d=\max _{j} d_{j}, \quad d_{j}=\operatorname{diam} \Omega_{j} \equiv \sup \left\{|x-y|: x, y \in \Omega_{j}\right\},
\end{gathered}
$$

а $c(\nu, s, \sigma)$ - некоторая константа, зависящая, вообще говоря, от $\Omega$. 
ДокАЗАТЕльство. Определим интерполящионный оператор $R_{\mathscr{T}}$, связанньй с набором $\mathscr{T}$, формулой

$$
\left(R_{\mathscr{T}} u\right)(x)=l_{j}(u)=\int_{\Omega_{j}} \lambda_{j}(x) u(x) d x, \quad x \in \Omega_{j}, \quad j=1, \ldots, N .
$$

Легко проверить, что

$$
\left\|u-R_{\mathscr{T}} u\right\| \leqslant c_{\nu} \cdot \Lambda \cdot\|u\|, \quad u \in L^{2}(\Omega) .
$$

Далее, из леммы 3.2 вытекает, что

$$
\left\|u-l_{j}(u)\right\|_{L^{2}\left(\Omega_{j}\right)} \leqslant \frac{\sigma_{\nu}}{\nu} d_{j}^{\nu+1}\|\lambda\|_{L^{\infty}\left(\Omega_{j}\right)}\|\nabla u\|_{L^{2}\left(\Omega_{j}\right)} .
$$

Отсюда получаем оценку

$$
\left\|u-R_{\mathscr{T}} u\right\| \leqslant \frac{\sigma_{\nu}}{\nu} \cdot d \cdot \Lambda \cdot\|u\|_{H^{1}(\Omega)} .
$$

Поэтому, используя тот факт, что $H^{s}(\Omega)$ при $0<s<1$ получается интерполяцией между $L^{2}(\Omega)$ и $H^{1}(\Omega)$, из (3.18) и (3.19) находим, что

$$
\left\|u-R_{\mathscr{T}} u\right\| \leqslant\left(c_{\nu} \cdot \Lambda\right)^{1-s} \cdot\left(\frac{\sigma_{\nu}}{\nu} d \Lambda\right)^{s} \cdot\|u\|_{s}
$$

для всех $0 \leqslant s \leqslant 1$, где, как и ранее, $\|\cdot\|_{s}$ - норма в пространстве $H^{s}(\Omega)$. Следовательно, теорема 2.3 дает соотношение

$$
\varepsilon_{\mathscr{L}}(s, 0)=\varepsilon_{\mathscr{L}}\left(H^{s}(\Omega), L^{2}(\Omega)\right) \leqslant c(\nu, s) \cdot \Lambda \cdot d^{s}, \quad 0 \leqslant s \leqslant 1 .
$$

Так как в случае сильно липшицевых областей сушествует (см. [31], [33]) непрерывньй оператор продолжения из $H^{s}(\Omega)$ в $H^{s}\left(\mathbb{R}^{\nu}\right), s \geqslant 0$, то можно установить (см., например, [34]) интерполящионные неравенства

$$
\|u\|_{s_{1} \theta+s_{2}(1-\theta)} \leqslant c\left(\theta, s_{1}, s_{2}\right) \cdot\|u\|_{s_{1}}^{\theta} \cdot\|u\|_{s_{2}}^{1-\theta}, \quad s_{1}, s_{2} \geqslant 0, \quad 0 \leqslant \theta \leqslant 1,
$$

где константа $c\left(\theta, s_{1}, s_{2}\right)$ зависит, вообще говоря, и от области $\Omega$. Эти неравенства позволяют воспользоваться леммой 2.1 и завершить доказательство теоремы.

Проиллюстрируем эту теорему следуюшим примером. 
ПримеР 3.1. Пусть $\Omega=(0, l)^{\nu}-$ куб в $\mathbb{R}^{\nu}$ с ребром длины $l$. Построим набор $\mathscr{T}=\left\{\left(\Omega_{j}, \lambda_{j}\right)\right\}$, задаюший локальные объемные средние, следуюшим образом. Пусть $K=(0,1)^{\nu}$ - стандартньй единичный куб в $\mathbb{R}^{\nu}$ и $\omega$ - некоторое измеримое множество в $K$ положительной лебеговой меры, mes $\omega>0$. Определим функцию $\lambda(\omega, x)$ на $K$ формулой

$$
\lambda(\omega, x)= \begin{cases}{[\operatorname{mes} \omega]^{-1},} & x \in \omega \\ 0, & x \in K \backslash \omega\end{cases}
$$

Положим

$$
\begin{gathered}
\Omega_{j}=(j+K) \cdot h \equiv\left\{x=\left(x_{1}, \ldots, x_{\nu}\right): j_{i}<\frac{x_{i}}{h}<j_{i}+1, i=1, \ldots, \nu\right\}, \\
\lambda_{j}(x)=\frac{1}{h^{\nu}} \lambda\left(\omega, \frac{x}{h}-j\right), \quad x \in \Omega_{j},
\end{gathered}
$$

для любого мультииндекса $j=\left(j_{1}, \ldots, j_{\nu}\right)$, где $j_{i}=0,1, \ldots, N-1, h=l / N$. Ясно, что для набора $\mathscr{T}=\left\{\left(\Omega_{j}, \lambda_{j}\right)\right\}$ вьполнены условия теоремы 3.1. При этом

$$
\Lambda=\frac{\nu^{\nu / 2}}{\operatorname{mes} \omega}, \quad d_{j}=\operatorname{diam} \Omega_{j}=\sqrt{\nu} \cdot h,
$$

и, следовательно, в данном случае для множества $\mathscr{L}$ вида (3.16) имеем, что

$$
\varepsilon_{\mathscr{L}}\left(H^{s}(\Omega), H^{\sigma}(\Omega)\right) \leqslant c(\nu, s, \sigma, \operatorname{mes} \omega, l) \cdot h^{s-\sigma}, \quad 0 \leqslant \sigma \leqslant s, \quad s \neq 0 .
$$

Отметим, что число функционалов, входящих в $\mathscr{L}$, в данном случае равно $\mathscr{N}_{\mathscr{L}}=N^{\nu}$. Поэтому оценка (3.22) может быть переписана в виде

$$
\varepsilon_{\mathscr{L}}\left(H^{s}(\Omega), H^{\sigma}(\Omega)\right) \leqslant c(\nu, s, \sigma, \operatorname{mes} \omega, l) \cdot\left(\frac{1}{\mathscr{N}_{\mathscr{L}}}\right)^{\frac{s-\sigma}{\nu}}, \quad 0 \leqslant \sigma \leqslant s, \quad s \neq 0 .
$$

С другой стороны, если в каждом пространстве $H^{s}(\Omega)$ рассмотреть подпространство $H_{\mathrm{per}}^{s}(\Omega) l$-периодических по каждой переменной $x_{i}$ функций:

$$
H_{\mathrm{per}}^{s}(\Omega)=\left\{u(x)=\sum_{k \in \mathbb{Z}^{\nu}} u_{k} e^{2 \pi i k x}: \sum_{k \in \mathbb{Z}^{\nu}}\left(1+|k|^{2}\right)^{s}\left|u_{k}\right|^{2}<\infty, u_{k}=\bar{u}_{-k}\right\}
$$

то, используя следствие 2.1, теорему 2.4 и явньй вид соответствуюшего оператора $K$, можно показать (см. также [29]-[31], где представлены аналогичные рассмотрения), что $\mathscr{N}_{\mathscr{L}}$-поперечник Колмогорова вложения $H_{\mathrm{per}}^{s}(\Omega)$ в $H_{\mathrm{per}}^{\sigma}(\Omega)$ имеет тот же порядок по $\mathscr{N}_{\mathscr{L}}$, т.е.

$$
\kappa_{\mathcal{N}_{\mathscr{L}}}\left(H_{\mathrm{per}}^{s}(\Omega), H_{\mathrm{per}}^{\sigma}(\Omega)\right)=c_{0} \cdot\left(\frac{1}{\mathscr{N}_{\mathscr{L}}}\right)^{\frac{s-\sigma}{\nu}}, \quad 0 \leqslant \sigma \leqslant s, \quad s \neq 0 .
$$

Таким образом, так как

$$
\varepsilon \mathscr{L}\left(H_{\mathrm{per}}^{s}(\Omega), H_{\mathrm{per}}^{\sigma}(\Omega)\right) \leqslant \varepsilon \mathscr{L}\left(H^{s}(\Omega), H^{\sigma}(\Omega)\right)
$$


то из теоремы 2.4 вытекает, что локальные объемные средние имеют дефект полноты, близкий (при фиксированном числе функционалов) к наилучшему. В рассматриваемом примере это приводит к двойному неравенству

$$
c_{1} \cdot h^{s-\sigma} \leqslant \varepsilon \mathscr{L}\left(H^{s}(\Omega), H^{\sigma}(\Omega)\right) \leqslant c_{2} \cdot h^{s-\sigma}, \quad 0 \leqslant \sigma \leqslant s, \quad s \neq 0,
$$

где $c_{1}$ и $c_{2}$ - некоторые положительные константы, которые могут зависеть от $s, \sigma, \nu$, $\operatorname{diam} \Omega, \operatorname{mes} \omega$. Аналогичное соотношение остается в силе и для областей более обшего вида (см. оценки поперечников, приведенные в [31]).

Дефект полноты для другого важного класса функционалов оценен в следуюшем утверждении.

ТеОРема 3.2. Предположим, что выполнены условия теоремы 3.1. Выберем в каждой подобласти $\Omega_{j}$ точку $x_{j}$ и определим на $H^{m}(\Omega), m=\left[\frac{\nu}{2}\right]+1$, множество функционалов (будем называть их узлами):

$$
\mathscr{L}=\left\{l_{j}(u)=u\left(x_{j}\right): x_{j} \in \Omega_{j}, j=1,2, \ldots, N\right\} .
$$

Тогда для всех $s \geqslant m u 0 \leqslant \sigma \leqslant s$ для дефекта полноты әтого множества функиионалов справедлива оценка

$$
\varepsilon_{\mathscr{L}}(s, \sigma) \equiv \varepsilon_{\mathscr{L}}\left(H^{s}(\Omega), H^{\sigma}(\Omega)\right) \leqslant c(\nu, s, \sigma) \cdot(\Lambda d)^{s-\sigma},
$$

где $\Lambda$ и d те же величины, что и в теореме 3.1, а $c(\nu, s, \sigma)$ - некоторая константа, зависящая, вообще говоря, от $\Omega$.

ДоКАЗАТЕльство. Пусть $u \in H^{m}(\Omega)$ и $l_{j}(u)=u\left(x_{j}\right)=0, j=1,2, \ldots, N$. Тогда, используя (3.12) для $\Omega=\Omega_{j}$ и $x_{j}=x_{*}$, получаем, что

$$
\|u\|_{L^{2}\left(\Omega_{j}\right)} \leqslant c \cdot \Lambda \sum_{l=1}^{m} d_{j}^{l} \sum_{|\alpha|=l} \frac{1}{\alpha !} \cdot\left\|D^{\alpha} u\right\|_{L^{2}\left(\Omega_{j}\right)} .
$$

Здесь и ниже $c$ - некоторая константа, меняюшаяся от формулы к формуле. Отсюда вытекает, что

$$
\|u\| \leqslant c \cdot \Lambda \sum_{l=1}^{m} d^{l} \cdot\|u\|_{l}
$$

для всех $u \in H^{m}(\Omega)$ таких, что $l_{j}(u)=u\left(x_{j}\right)=0, j=1,2, \ldots, N$. Пользуясь интерполящионным неравенством (3.21), находим, что

$$
\|u\| \leqslant c \cdot \Lambda \sum_{l=1}^{m} d^{l} \cdot\|u\|^{1-l / m} \cdot\|u\|_{m}^{l / m} .
$$

Так как

$$
\Lambda d^{l} \cdot\|u\|^{1-l / m} \cdot\|u\|_{m}^{l / m} \leqslant\left(1-\frac{l}{m}\right) \delta^{\frac{m}{m-l}}\|u\|+\frac{l}{m} \delta^{-\frac{m}{l}} \Lambda^{\frac{m}{l}} d^{m}\|u\|_{m}
$$


для всех $\delta>0$ и $l=1,2, \ldots, m-1$, то из (3.26) получаем, что

$$
\left(1-c_{\nu} \sum_{l=1}^{m-1}\left(1-\frac{l}{m}\right) \delta^{\frac{m}{m-l}}\right)\|u\| \leqslant c\left(\Lambda+\sum_{l=1}^{m-1} \frac{l}{m}\left(\frac{\Lambda}{\delta}\right)^{\frac{m}{l}}\right) d^{m}\|u\|_{m} .
$$

Выбирая подходящим образом $\delta=\delta(\nu, m)$, отсюда находим, что

$$
\|u\| \leqslant c \sum_{l=1}^{m} \frac{l}{m} \cdot \Lambda^{\frac{m}{l}} \cdot d^{m} \cdot\|u\|_{m}
$$

для всех $u \in H^{m}(\Omega)$ таких, что $l_{j}(u)=u\left(x_{j}\right)=0, j=1,2, \ldots, N$. Следовательно,

$$
\varepsilon \mathscr{L}\left(H^{m}(\Omega), L^{2}(\Omega)\right) \leqslant c d^{m} \sum_{l=1}^{m} \frac{l}{m} \Lambda^{\frac{m}{l}} .
$$

Так как $\Lambda \geqslant 1$, то отсюда вытекает неравенство (3.25) при $\sigma=0$ и $s=m=\left[\frac{\nu}{2}\right]+1$. Дальнейшие рассуждения, как и в случае теоремы 3.1, опираются на лемму 2.1 и интерполящионные неравенства (3.21). Теорема 3.2 доказана.

ПримеР 3.2. Вернемся к ситуации, описанной в примере 3.1. Положим $\omega=K$ и выберем узлы $x_{j} \in \Omega_{j}$. Тогда для множества $\mathscr{L}$ функционалов вида $(3.24)$ имеем, что

$$
\varepsilon_{\mathscr{L}}\left(H^{s}(\Omega), H^{\sigma}(\Omega)\right) \leqslant c(\nu, s, \sigma, l) \cdot h^{s-\sigma}
$$

для всех $s \geqslant\left[\frac{\nu}{2}\right]+1,0 \geqslant \sigma \geqslant s$ и любого расположения узлов $x_{j}$ внутри $\Omega_{j}$. В рассматриваемом случае сохраняется также двусторонняя оценка вида (3.23).

ЗАмечАниЕ 3.1. Соотношения (3.17) и (3.25) верны и для шкалы пространств $H_{0}^{s}(\Omega)$. Однако в этом случае нормы в $H_{0}^{s}(\Omega)$ можно выбрать так, чтобы константа $c(\nu, s, \sigma)$, фигурируюшая в (3.17) и (3.25), не зависела от $\Omega$. Это объясняется тем, что продолжение нулем вне $\Omega$ задает ограниченный оператор из $H_{0}^{s}(\Omega)$ в $H^{s}\left(\mathbb{R}^{\nu}\right)$ с оценкой нормы, не зависящей от $\Omega$, и поэтому константа $c(\nu, s, \sigma)$ в интерполяционных неравенствах (3.21) не зависит от $\Omega$. Разумеется, данное замечание остается справедливым во всех случаях, когда удается построить оператор продолжения из $H^{s}(\Omega)$ в $H^{s}\left(\mathbb{R}^{\nu}\right)$ с оценкой нормы, не зависящей от области.

ЗАмечАнИЕ 3.2. Пусть $\mathscr{L}$ - семейство функционалов, определенное на $H^{s}(\Omega)$ соотношениями (3.24). Теорема 3.2 , а также замечание 2.1 позволяют при каждом допустимом $s>0$ построить набор функций $\phi_{j}(x) \in H^{s}(\Omega)$ так, чтобы оператор $R_{\mathscr{L}}$, действующий в $H^{s}(\Omega)$ по формуле

$$
\left(R_{\mathscr{L}} u\right)(x)=\sum_{j=1}^{N} u\left(x_{j}\right) \phi_{j}(x)
$$

являлся интерполяционным лагранжевым $\left(\phi_{j}\left(x_{i}\right)=\delta_{j i}\right)$ оператором с оценкой погрешности интерполящии, совпадаюшей с соответствуюшим дефектом полноты. Аналогичное утверждение справедливо и для случая локальных объемных средних, построенных в теореме 3.1. К сожалению (см., замечание 2.1 и доказательство теоремы 2.3), с точки зрения практических вычислений задание функций $\phi_{j}(x)$ не является явным. 


\section{$\S 4$. Определяющие функционалы для полулинейных параболических уравнений}

В этом параграфе рассматривается вопрос о сушествовании и свойствах определяющих функционалов для задач, порождаемых в некотором сепарабельном гильбертовом пространстве $H$ уравнением вида

$$
\frac{d u}{d t}+A u=B(u, t), \quad t>0,\left.\quad u\right|_{t=0}=u_{0} \in H
$$

Здесь $A$-положительньй оператор с дискретньм спектром, т.е. сушествует ортонормированньй базис $\left\{e_{i}\right\}$ в пространстве $H$, состояший из собственных векторов оператора $A$ :

$$
A e_{i}=\lambda_{i} e_{i}, \quad\left(e_{i}, e_{j}\right)_{H}=\delta_{i j}, \quad 0<\lambda_{1} \leqslant \lambda_{2} \leqslant \cdots, \quad \lim _{N \rightarrow \infty} \lambda_{N}=\infty
$$

а функция $B(u, t)$ при некотором $0 \leqslant \gamma<1 / 2$ является непрерьвным отображением из $D\left(A^{1 / 2}\right) \times \mathbb{R}$ в пространство $D\left(A^{-\gamma}\right)$ и обладает свойством

$$
\left\|A^{-\gamma}\left(B\left(u_{1}, t\right)-B\left(u_{2}, t\right)\right)\right\| \leqslant M(\rho)\left\|A^{1 / 2}\left(u_{1}-u_{2}\right)\right\|
$$

для всех $u_{j} \in D\left(A^{1 / 2}\right)$ таких, что $\left\|A^{1 / 2} u_{j}\right\| \leqslant \rho$, где $\rho$-произвольное положительное число, $M(\rho)>0$ - некоторая константа, $\|\cdot\|=\|\cdot\|_{H}-$ норма в $H$. Здесь $D\left(A^{-\gamma}\right)-$ пополнение $H$ по норме $\left\|A^{-\gamma} \cdot\right\|$.

Предположим, что задача (4.1) однозначно разрешима в классе функций

$$
\mathscr{W}=C([0,+\infty) ; H) \cap C\left((0,+\infty) ; D\left(A^{1 / 2}\right)\right)
$$

и является точечно диссипативной, т.е. существует $R>0$ такое, что

$$
\left\|A^{1 / 2} u(t)\right\| \leqslant R \text { при } t \geqslant t_{0}(u)
$$

для всех $u(t) \in \mathscr{W}$. Конкретные примеры задач вида (4.1), для которых вьполнены приведенные выше свойства, можно найти, например, в [1]-[5]. Этому классу задач принадлежат, в частности, некоторые уравнения реакции-диффузии, двумерная система Навье-Стокса, двумерные уравнения магнитной гидродинамики.

Результаты, полученные в $\S 1$ и $\S 2$, позволяют установить следуюшее утверждение.

ТеОРема 4.1. Предположим, что выполнены условия (4.2) и (4.4). Пусть $\mathscr{L}=\left\{l_{j}: j=1, \ldots, N\right\}-$ множество линейных непрерывных функционалов на пространстве $V=D\left(A^{1 / 2}\right)$, снабжсенном нормой $\|\cdot\|_{V}=\left\|A^{1 / 2} \cdot\right\|$. Тогда $\mathscr{L}$ является множеством асимптотически $(V, H, \mathscr{W})$-определяющих функиионалов задачи (4.1) при условии, что дефект полноты $\varepsilon_{\mathscr{L}}(V, H)$ удовлетворяет неравенству

$$
\varepsilon \mathscr{L}(V, H)<\varepsilon_{0}(\gamma, R) \equiv \sqrt{\frac{1+2 \gamma}{1-2 \gamma}} \cdot[(1+2 \gamma) M(R)]^{-\frac{1}{1-2 \gamma}},
$$

где $M(\rho)$ и $R$ - величинь, фигурирующие в (4.2) и (4.4). 
ДоКАЗАТЕльство. Для любой пары решений $u(t), v(t) \in \mathscr{W}$ задачи $(4.1)$, используя (4.1) и (4.2), легко обнаружить, что

$$
\frac{1}{2}\|w(t)\|^{2}+\int_{s}^{t}\left\|A^{1 / 2} w(\tau)\right\|^{2} d \tau \leqslant \frac{1}{2}\|w(s)\|^{2}+M(R) \int_{s}^{t}\left\|A^{1 / 2} w(\tau)\right\| \cdot\left\|A^{\gamma} w(\tau)\right\| d \tau
$$

при $t \geqslant s$, где $s \geqslant 0$ достаточно велико и $w(t)=u(t)-v(t)$. Отсюда, пользуясь очевидньм соотношением $\left\|A^{\gamma} w\right\| \leqslant\left\|A^{1 / 2} w\right\|^{2 \gamma} \cdot\|w\|^{1-2 \gamma}$ и неравенством Гёльдера $x y \leqslant x^{p} / p+y^{q} / q, p^{-1}+q^{-1}=1$, с подходяшими $p, q>1$, получаем, что

$$
\begin{aligned}
M(R)\left\|A^{1 / 2} w\right\| \cdot\left\|A^{\gamma} w\right\| & \leqslant M(R)\left\|A^{1 / 2} w\right\|^{1+2 \gamma} \cdot\|w\|^{1-2 \gamma} \\
& \leqslant \frac{1}{2}\left\|A^{1 / 2} w\right\|^{2}+\frac{1}{2} \varepsilon_{0}(\gamma, R)^{-2}\|w\|^{2} .
\end{aligned}
$$

Поэтому

$$
\|w(t)\|^{2}+\int_{s}^{t}\left\|A^{1 / 2} w(\tau)\right\|^{2} d \tau \leqslant\|w(s)\|^{2}+\varepsilon_{0}(\gamma, R)^{-2} \int_{s}^{t}\|w(\tau)\|^{2} d \tau .
$$

Теперь утверждение теоремы 4.1 вытекает из следствия 1.1 и теоремы 2.1.

Теоремы 4.1 и 2.4 позволяют легко получить условия сушествования $N$ определяюших функшионалов.

СлЕДСТВИЕ 4.1. Предположим, что $N$-поперечник Колмогорова $\kappa_{N}(V, H)$ вложения пространства $V=D\left(A^{1 / 2}\right)$ в $H$ обладает свойством $\kappa_{N}(V, H)<$ $\varepsilon_{0}(\gamma, R)$, где $\varepsilon_{0}(\gamma, R)$ - величина, фигурирующая в (4.5). Тогда существует множество $\mathscr{L}$ асимптотически $(V, H, \mathscr{W})$-определяющих функционалов задачи (4.1), состоящее из $N$ әлементов. $B$ частности, в качестве $\mathscr{L}$ можно взять множество определяющих мод:

$$
\mathscr{L}=\left\{l_{j}(v)=\left(v, e_{j}\right), j=1,2, \ldots, N\right\} .
$$

Здесь $\left\{e_{j}\right\}$ - собственнье векторы оператора $A$, упорядоченные по возрастанию собственных чисел. При этом $\kappa_{N}(V, H)=\lambda_{N+1}^{-1 / 2}$.

ЗАмЕчАнИЕ 4.1. Используя свойство сглаживания решений для параболических задач вида (4.1) и соотношение (4.4), легко проверить, что в условиях теоремы 4.1 $\mathscr{L}$ является множеством асимптотически $(V, V, \mathscr{W})$-определяюших функционалов, т.е. сближение траекторий происходит в более сильной норме. Кроме того, оно является также определяющим в следуюшем смысле (ср. с определением 1.2): для любой пары решений $u_{1}(t)$ и $u_{2}(t)$ задачи $(4.1)$, определенных на всей временной оси и таких, что

$$
\sup \left\{\left\|A^{1 / 2} u_{i}(t)\right\|:-\infty<t<\infty\right\} \leqslant R, \quad i=1,2,
$$

из условия $l_{j}\left(u_{1}(t)\right)=l_{j}\left(u_{2}(t)\right)$ для почти всех $t \in \mathbb{R}$ и $j=1, \ldots, N$ вытекает, что $u_{1}(t) \equiv u_{2}(t)$. Доказательство последнего утверждения вытекает из (4.6) и следствия 1.2. Очевидно также, что в том случае, когда условие (4.2) вьполняется с константой $M$, не зависящей от $\rho$, от требования диссипативности (4.4) в теореме 4.1 можно избавится. 
ЗАмЕчАниЕ 4.2. При дополнительных условиях (см. [2], [23]-[26]), наиболее ограничительным из которых является требование достаточно быстрого роста величины $\lambda_{N+1}-\lambda_{N}$ при $N \rightarrow \infty$, задача (4.1) обладает асимптотически полным инерциальньм многообразием $\mathscr{M}_{t}$ вида $(1.27)$, где $V=D\left(A^{\theta}\right)$ при некотором $0<\theta<1$, а $P=P_{N}$ - ортопроектор на первые $N$ собственных векторов оператора $A$ ( $N$ достаточно велико). При этом константа Липшица $L$ (см. (1.28)) не зависит от $N$ и вьполняются все условия теоремы 1.3 с $\Lambda=\lambda_{N}^{\theta}$. Поэтому соотношение вида $\varepsilon \mathscr{L}(V, H)<c_{0} \lambda_{N}^{-\theta}$ гарантирует выполнение заключений теоремы 1.3 в рассматриваемой здесь ситуации.

ПримеР 4.1 (система Навье-Стокса). Рассмотрим уравнения, описывающие динамику вязкой несжимаемой жидкости, заполняющей область $\Omega \equiv T^{2}=(0, L) \times(0, L)$, с периодическими граничными условиями:

$$
\begin{gathered}
\partial_{t} u-\nu \Delta u+(u, \nabla) u+\nabla p=F(x, t), \quad x \in T^{2}, \quad t>0, \\
\nabla u=0, \quad x \in T^{2}, \quad u(x, 0)=u_{0}(x),
\end{gathered}
$$

где неизвестные вектор скорости $u(x, t)=\left(u_{1}(x, t) ; u_{2}(x, t)\right)$ и давление $p(x, t)$ являются $L$-периодическими функциями по пространственньм переменным, $\nu>0, F(x, t)$ - внешняя сила.

Введем некоторые определения. Пусть $\mathscr{V}$ - пространство тригонометрических полиномов $v(x)$ периода $L$ со значениями в $\mathbb{R}^{2}$ таких, что $\operatorname{div} v=0$ и $\int_{T^{2}} v(x) d x=0$. Пусть $H$ - замькание $\mathscr{V}$ в $\mathbf{L}^{2}=L^{2}(\Omega) \times L^{2}(\Omega), \Pi$ - ортогональньй проектор в $\mathbf{L}^{2}$ на $H, A=-\Pi \Delta u=-\Delta u, B(u, v)=\Pi(u, \nabla) v$ для всех $u$ и $v$ из $D(A)=H \cap \mathbf{H}^{2}$, где $\mathbf{H}^{2}=H_{\text {per }}^{2}(\Omega) \times H_{\text {per }}^{2}(\Omega)$. Напомним (см. [35], [36]), что $A$-положительньй оператор с дискретным спектром, а билинейный оператор $B(u, v)$ дает непрерывное отображение из $D(A) \times D(A)$ в $H$ и из $D\left(A^{1 / 2}\right) \times D\left(A^{1 / 2}\right)$ в $D\left(A^{-\gamma}\right)$ при любом $\gamma>0$. Задача $(4.7)$ при этом запишется в виде

$$
\partial_{t} u+\nu A u+B(u, u)=\Pi F(t),\left.\quad u\right|_{t=0}=u_{0} \in H .
$$

Хорошо известно (см., например, [35], [36]), что если $u_{0} \in H$ и $\Pi F(t) \in L^{\infty}\left(\mathbb{R}_{+} ; H\right)$, то у задачи (4.8) сушествует единственное решение $u(t)$ такое, что

$$
u(t) \in \mathscr{W}=C\left(\mathbb{R}_{+} ; H\right) \cap C\left(t_{0},+\infty ; D(A)\right), \quad t_{0}>0 .
$$

Можно показать [8], [36], что оно обладает свойствами

$$
\varlimsup_{t \rightarrow \infty}\left\|A^{1 / 2} u(t)\right\|^{2} \leqslant \frac{F^{2}}{\nu^{2} \lambda_{1}}
$$

и

$$
\varlimsup_{t \rightarrow \infty} \frac{1}{a} \int_{t}^{t+a}\|A u(\tau)\|^{2} d \tau \leqslant \frac{F^{2}}{\nu^{2}} \cdot\left(1+\frac{1}{a \nu \lambda_{1}}\right)
$$

для любого $a>0$. Здесь $\lambda_{1}=(2 \pi / L)^{2}$ - первое собственное число оператора $A$ в $H$, $F=\varlimsup_{t \rightarrow \infty}\|\Pi F(t)\|$. При этом рассмотрения, приведенные в [8, с. 177-178], дают, что если $\Pi F(t) \in L^{\infty}\left(\mathbb{R}_{+} ; D\left(A^{1 / 2}\right)\right)$, то

$$
\varlimsup_{t \rightarrow \infty}\|A u(t)\|^{2} \leqslant\left(1+\frac{c_{0} F^{2}}{\nu^{4} \lambda_{1}}\right) \cdot \frac{F^{2}}{\nu^{2}}+\frac{2 F_{1}^{2}}{\nu^{2} \lambda_{1}} \equiv D,
$$


где $F_{1}=\varlimsup_{t \rightarrow \infty}\|\nabla \Pi F(t)\|, c_{0}$ - некоторая абсолютная константа.

Из непрерьвности вложений $H^{1-\delta}(\Omega)$ в $L^{2 / \delta}(\Omega)$ и $L^{2 /(1+\delta)}(\Omega)$ в $H^{-\delta}(\Omega)$ при $0<\delta<1$ и неравенства Гёльдера получаем, что

$$
\begin{aligned}
\|(u, \nabla) v\|_{\mathbf{H}^{-\delta}(\Omega)} & \leqslant C\|(u, \nabla) v\|_{\mathbf{L}^{2 /(1+\delta)}(\Omega)} \\
& \leqslant C\|u\|_{\mathbf{L}^{2 / \delta}(\Omega)}\|\nabla v\|_{\mathbf{L}^{2}(\Omega)} \\
& \leqslant C\|u\|_{\mathbf{H}^{1-\delta}(\Omega)}\|v\|_{\mathbf{H}^{1}(\Omega)} .
\end{aligned}
$$

Отсюда и из (4.10) вытекает, что задача (4.8) удовлетворяет условиям теоремы 4.1. Поэтому любое множество $\mathscr{L}$ функционалов на $V=D\left(A^{1 / 2}\right)=H \cap \mathbf{H}^{1}$ является асимптотически $(V, H, \mathscr{W})$-определяюшим при достаточно малом дефекте $\varepsilon \mathscr{L}(V, H)$. При этом не трудно обнаружить, что оценка (4.5) в данном случае приобретает вид

$$
\left[\varepsilon_{\mathscr{L}}(V, H)\right]^{1-\delta}<C_{\delta} \cdot L^{2-\delta}(\nu G)^{-1},
$$

где $G=F \cdot\left(\lambda \nu^{2}\right)^{-1}$ - обобщенное число Грасгофа (оно было введено в [8]), $\delta$ - любое число из интервала $(0,1), C_{\delta}$ - константа, зависяшая только от $\delta$. А так как для собственных чисел $\lambda_{N}$ оператора Стокса $A=-\Pi \Delta$ на торе $T^{2}$ выполняется свойство (см., например, [35], [36]):

$$
\alpha N \lambda_{1} \leqslant \lambda_{N} \leqslant \beta N \lambda_{1}
$$

где $\alpha, \beta>0$ - некоторые абсолютные константы, то следствие 4.1 дает, что задача (4.7) обладает набором из $N$ определяюших функционалов для каждого $N$, удовлетворяющего оценке вида $N^{1-\delta} \geqslant c_{\delta} \cdot((\nu / L) G)^{2}$. В частности (см. теорему 3.1 и пример 3.1), если $N$ подчиняется этой оценке, то задача (4.7) имеет $N$ определяющих обобщенных локальных объемных средних. Однако, ввиду того, что $D\left(A^{1 / 2}\right) \not \subset C(\Omega)$, теорема 4.1 не гарантирует сушествования определяюших узлов в задаче (4.7). В этой связи представляет интерес приведенная ниже теорема 4.2, которая, с одной стороны, позволяет использовать менее гладкие функционалы, а с другой, дает более слабые условия на дефект и число функционалов. Разумеется, эта теорема существенно опирается на структуру задачи (4.7).

Tеорема 4.2. 1. Множество $\mathscr{L}=\left\{l_{j}: j=1, \ldots, N\right\}$ линейно независимых функиионалов на $D(A)=\mathbf{H}^{2} \cap H$ является асимптотически $\left(D(A), D\left(A^{1 / 2}\right), \mathscr{W}\right)$-определяющим для задачи $(4.8)$, где $\mathscr{W}$ определяется равенством (4.9), если $\varepsilon \mathscr{L}(D(A), H)<c_{1} \nu^{2} F^{-1} \equiv c_{1}\left(\lambda_{1} G\right)^{-1}$, где $c_{1}$ - абсолютная константа, $G=F \cdot\left(\lambda \nu^{2}\right)^{-1}-$ обобщенное число Грасгофа.

2. Пусть $\mathscr{L}=\left\{l_{j}: j=1, \ldots, N\right\}$ - множество линейных функционалов на $H^{2}(\Omega)$, и пусть $p_{m}$ - естественная проекция вектора скорости на направление $m=\left(m_{1} ; m_{2}\right): p_{m}\left(u_{1} ; u_{2}\right)=(m, u) \equiv m_{1} u_{1}+m_{2} u_{2}$, вектор $m$ не зависит от $x$, $m_{1}^{2}+m_{2}^{2}=1$. Определим множество $p_{m}^{*} \mathscr{L}$ функционалов на $D(A)$ формулой

$$
p_{m}^{*} \mathscr{L}=\left\{l_{j}^{m}(u)=l_{j}\left(p_{m} u\right): l_{j} \in \mathscr{L}, u \in D(A), j=1, \ldots, N\right\} .
$$

Предположим, что $\Pi F(t) \in L^{\infty}\left(\mathbb{R}_{+} ; D\left(A^{1 / 2}\right)\right)$. Пусть $\widehat{H}-$ подпространство в $L^{2}(\Omega)$, состоящее из функций, обладающих свойством $\int_{\Omega} v d x=0, u \widehat{H}^{2}=$ $H_{\mathrm{per}}^{2}(\Omega) \cap \widehat{H}$, причем $\widehat{H}^{2}$ снабәсено нормой $\|\Delta \cdot\|$. Тогда если

$$
\widehat{\varepsilon}_{\mathscr{L}} \equiv \varepsilon_{\mathscr{L}}\left(\widehat{H}^{2}, \widehat{H}\right)<c_{2} \nu^{2} L^{-2} D^{-1},
$$


где $c_{2}$ - некоторая абсолютная константа, а величина $D$ определена в (4.12), то для каждого орта $m=\left(m_{1} ; m_{2}\right)$ множество $p_{m}^{*} \mathscr{L}$ является асимптотически $\left(D(A), D\left(A^{1 / 2}\right), \mathscr{W}\right)$-определяющим для задачи (4.8).

ДокАЗАТЕльство первого утверждения этой теоремы опирается на лемму 1.1 и соотношение (4.11). Фактически оно хорошо известно (см. [6], [8], [10], [13], [14], где рассмотрены конкретные семейства определяюших функционалов и приведены различные оценки их числа, а также [17], [20], где анализируется случай функционалов общего вида).

Заметим, что соотношение (4.13) и теорема 2.4 о связи дефекта полноты и поперечника Колмогорова позволяют из первого утверждения теоремы 4.2 извлечь существование у задачи (4.7) конечного набора определяюших мод с числом элементов порядка $G$. Благодаря теоремам 3.1 и 3.2 система (4.7) обладает конечными определяюшими семействами обобшенных локальных объемных средних и узлов с числом элементов того же порядка $G$ (см. также [14]). Этот результат согласуется с эвристическими оценками, полученными ранее на основе физических соображений (см. обсуждение в [8], [14] и ссылки, приведенные в этих работах). Следует отметить, что для двумерной системы Навье-Стокса на торе обобщенное число Грасгофа $G$ играет роль, аналогичную числу Рейнольдса, и является естественным параметром, характеризуюшим сложность динамики (см., например, [8], [14], [36]).

Что касается второго утверждения теоремы 4.2, то его доказательство использует следующую лемму.

Лемма 4.1. Пусть $u, v \in D(A) u w=u-v$. Тогда для любого постоянного вектора $m=\left(m_{1} ; m_{2}\right)$ такого, что $m_{1}^{2}+m_{2}^{2}=1$, справедлива оценка

$$
|(B(u, u)-B(v, v), A w)| \leqslant c L\|\nabla(m, w)\|\|A w\|\|A u\|,
$$

где $(m, w)=m_{1} w_{1}+m_{2} w_{2}-$ проекиия вектора $w$ на направление $m, c$ - некоторая абсолютная константа.

ДокАЗАТЕЛЬСТво. Используя тождество (см. [36])

$$
(B(u, w), A w)+(B(w, u), A w)+(B(w, w), A u)=0
$$

справедливое для любых $u, w \in D(A)$, легко обнаружить, что

$$
(B(u, u)-B(v, v), A w)=-(B(w, w), A u),
$$

где $u, v \in D(A), w=u-v$. Поэтому достаточно оценить норму $\|B(w, w)\|$. Условие несжимаемости $\nabla w=0$ и простые вычисления дают, что

$$
\begin{aligned}
|(w(x), \nabla) w(x)|^{2}=( & (m, w(x))^{2}|\nabla w(x)|^{2}+|w(x)|^{2}|\nabla(m, w(x))|^{2} \\
& -\frac{1}{2}\left(\partial_{2}\left[(m, w(x))^{2}\right] \partial_{2}|w(x)|^{2}+\partial_{1}\left[(m, w(x))^{2}\right] \partial_{1}|w(x)|^{2}\right) .
\end{aligned}
$$

Отсюда вытекает, что

$$
|(w(x), \nabla) w(x)|^{2} \leqslant 2(m, w(x))^{2}|\nabla w(x)|^{2}+2|w(x)|^{2}|\nabla(m, w(x))|^{2} .
$$


Следовательно,

Поэтому

$$
\|(w, \nabla) w\|^{2} \leqslant 2\left(\|(m, w) \nabla w\|^{2}+\|w \nabla(m, w)\|^{2}\right) .
$$

$$
\|(w, \nabla) w\| \leqslant \sqrt{2}\left(\|(m, w)\|_{L^{4}}\|\nabla w\|_{L^{4}}+\|w\|_{L^{\infty}}\|\nabla(m, w)\|\right) .
$$

Следовательно, оценка (4.15) вытекает из неравенств

$$
\|v\|_{L^{4}} \leqslant c_{1} L^{1 / 2}\|\nabla v\|, \quad\|v\|_{L^{\infty}} \leqslant c_{2} L\|\Delta v\|,
$$

справедливых в рассматриваемом классе функций (см. [35], [36], а также работу [8], содержашую явные выражения для $c_{1}$ и $c_{2}$ ).

ДокАЗАТЕЛЬСТВо П. 2 тЕОРемЫ 4.2. Пусть $u(t)$ и $v(t)$ - решения задачи (4.8), обладаюшие свойством (4.9). Тогда из (4.8) и (4.15) для $w=u-v$ имеем, что

$$
\frac{1}{2} \frac{d}{d t}\|\nabla w\|^{2}+\nu\|\Delta w\|^{2} \leqslant c L\|\nabla(m, w)\|\|A w\|\|A u\| .
$$

Отсюда и из (4.12) вытекает, что

$$
\frac{d}{d t}\|\nabla w\|^{2}+\nu\|\Delta w\|^{2} \leqslant c D L^{2} \nu^{-1}\|\nabla(m, w)\|^{2}
$$

для достаточно больших $t$. Теорема 2.1 дает оценку

$$
\|\phi\| \leqslant C \eta(\phi)+\widehat{\varepsilon}_{\mathscr{L}}\|\Delta \phi\|, \quad \eta(\phi)=\max _{j}\left|l_{j}(\phi)\right|, \quad \phi \in \widehat{H}^{2} .
$$

Поэтому неравенство

$$
\|\nabla(m, w)\|^{2} \leqslant\|(m, w)\| \cdot\|\Delta(m, w)\|
$$

позволяет получить соотношение

$$
\|\nabla(m, w)\|^{2} \leqslant C_{\delta, \mathscr{L}} \eta((m, w))^{2}+(1+\delta) \widehat{\varepsilon}_{\mathscr{L}} \cdot\|\Delta(m, w)\|^{2}
$$

для каждого $\delta>0$. Кроме того, очевидно, что

$$
\|\Delta w\|^{2}=\|\Delta(m, w)\|^{2}+\left\|\Delta\left(m^{\perp}, w\right)\right\|^{2},
$$

где $m^{\perp}=\left(m_{2} ;-m_{1}\right)$. Поэтому из $(4.16)$ вытекает, что

$$
\frac{d}{d t}\|\nabla w(t)\|^{2}+\left[\nu-c(1+\delta) \widehat{\varepsilon}_{\mathscr{L}} D L^{2} \nu^{-1}\right] \cdot\|\Delta w\|^{2} \leqslant C_{\delta} \eta((m, w))^{2}
$$

для достаточно больших $t$. Следовательно, условие (4.14) с подходящей константой $c_{2}$ дает возможность воспользоваться теоремой 1.1 и получить второе утверждение теоремы 4.2 .

Теорема 4.2 , в частности, означает, что только один компонент вектора скорости является существенным для однозначного определения асимптотического поведения решений задачи (4.7). Однако, условие (4.14) на дефект полноты приводит к необходимости рассматривать в данной ситуации значительно большее число функционалов по сравнению со случаем утверждения 1 теоремы 4.2. Поэтому этот результат следует рассматривать, в основном, как утверждение качественного характера, свидетельствуюшее о том, что некоторые компоненты вектора состояний могут оказаться не существенньми с точки зрения асимптотического поведения. Более подробно данный эффект обсуждается в работе [20] (см. также [19]). В статье [20] для систем уравнений реакции-диффузии обшего вида приведены условия, при которых удается выделять несущественные для асимптотической динамики компоненты вектора решений. Эти условия вьполняются для довольно широкого класса систем. Например, справедливо следуюшее утверждение, относящееся к задаче (4.1). 
Теорема 4.3. 1. Предположим, что задача (4.1) разрешима в классе функиий

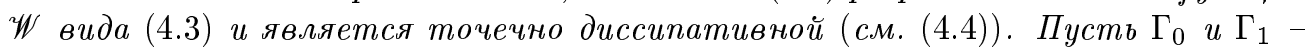
гильбертовы пространства с нормами $\|\cdot\|_{\Gamma_{0}} u\|\cdot\|_{\Gamma_{1}}$ такие, что $\Gamma_{1} \subset \Gamma_{0}$. Предположим, что существует линейный оператор $J: V=D\left(A^{1 / 2}\right) \mapsto \Gamma_{1}$ такой, что $\|J u\|_{\Gamma_{1}} \leqslant K \cdot\|u\|_{V} u$ (вместо (4.2)) выполняется неравенство

$$
\frac{1}{2}\left\|A^{1 / 2}\left(u_{1}-u_{2}\right)\right\|^{2}-\left(B\left(u_{1}, t\right)-B\left(u_{2}, t\right), u_{1}-u_{2}\right) \geqslant-C(R)\left\|J\left(u_{1}-u_{2}\right)\right\|_{\Gamma_{0}}^{2}
$$

для всех $u_{j} \in D\left(A^{1 / 2}\right)$, обладаюших свойством $\left\|A^{1 / 2} u_{j}\right\| \leqslant R$, где $R>0$ - константа из (4.4), $C(R)$ - некоторое положительное число. Здесь $(\cdot, \cdot)$ - скалярное произведение в $H$. Пусть $\mathscr{L}=\left\{l_{i}: i=1, \ldots, N\right\}$ - множсество линейных непрерывных функиионалов на $\Gamma_{1}$. Тогда

$$
J^{*} \mathscr{L}=\left\{l_{i}^{J}(w)=l_{i}(J w): l_{i} \in \mathscr{L}\right\}
$$

является асимптотически $(V, H, \mathscr{W})$-определяющим множеством для задачи (4.1) при условии, что $\varepsilon \mathscr{L}\left(\Gamma_{1}, \Gamma_{0}\right)<\left(2 C(R) K^{2}\right)^{-1 / 2}$.

ДокАЗАТЕЛЬСтво. Легко обнаружить, что

$$
\frac{1}{2} \frac{d}{d t}\|w(t)\|^{2}+(A w(t), w(t))=\left(B\left(u_{1}(t), t\right)-B\left(u_{2}(t), t\right), w(t)\right)
$$

для разности $w(t)=u_{1}(t)-u_{2}(t)$ двух решений $u_{1}(t)$ и $u_{2}(t)$ из $\mathscr{W}$. Следовательно, условия теоремы дают, что

$$
\frac{d}{d t}\|w(t)\|^{2}+\left\|A^{1 / 2} w(t)\right\|^{2} \leqslant 2 C(R)\|J w(t)\|_{\Gamma_{0}}^{2}
$$

для достаточно больших $t$. Поэтому из теоремы 2.1 вытекает, что

$$
\frac{d}{d t}\|w(t)\|^{2}+\omega \cdot\left\|A^{1 / 2} w(t)\right\|^{2} \leqslant C_{\mathscr{L}, \eta} \cdot \max _{i}\left|l_{i}(J w(t))\right|^{2}
$$

для достаточно больших $t$, где $\omega=1-2(1+\eta) \varepsilon_{\mathscr{L}}^{2}\left(\Gamma_{1}, \Gamma_{0}\right) C(R) K^{2}$ с произвольным $\eta>0$. Отсюда вытекает утверждение теоремы 4.3.

В качестве приложения теоремы 4.3 рассмотрим задачу о передаче нервных импульсов (о других ее применениях см. $\S 6$ ).

ПРИмеР 4.2. Рассматривается следуюшая система уравнений, предложенная Ходжкиным и Хаксли для описания механизма передачи нервных импульсов:

$$
\begin{gathered}
\partial_{t} u-d_{0} \partial_{x}^{2} u+g\left(u, v_{1}, v_{2}, v_{3}\right)=0, \quad x \in(0, L), \quad t>0 \\
\partial_{t} v_{j}-d_{j} \partial_{x}^{2} u+k_{j}(u) \cdot\left(v_{j}-h_{j}(u)\right)=0, \quad x \in(0, L), \quad t>0 .
\end{gathered}
$$

Здесь $d_{0}>0, d_{j} \geqslant 0, j=1,2,3$, и

$$
g\left(u, v_{1}, v_{2}, v_{3}\right)=-\gamma_{1} v_{1}^{3} v_{2}\left(\delta_{1}-u\right)-\gamma_{2} v_{3}^{4}\left(\delta_{2}-u\right)-\gamma_{3}\left(\delta_{3}-u\right),
$$


где $\gamma_{j}>0$ и $\delta_{1}>\delta_{3}>0>\delta_{2}$. Предполагается, что $k_{j}(u)$ и $h_{j}(u)$ - заданные непрерьвно дифференцируемые функции такие, что $k_{j}(u)>0$ и $0<h_{j}(u)<1, j=1,2,3$. В этой модели $u$ имеет смысл электрического потенциала в нерве, а $v_{j}$ являются коцентрациями химических веществ и могут изменяться между 0 и 1 . При различных граничных условиях задача $(4.17),(4.18)$ изучалась многими авторами (см., например, [2], [37]-[39] и литературу, цитируемую в этих работах). Результаты численного моделирования, представленные в [37], показьвают, что асимптотическое поведение решений этой задачи может быть довольно сложным. Ниже показано, что асимптотическое поведение концентраций $v_{j}$ однозначно определяется множествами функционалов, заданными лишш на электрическом потенциале $u$. Таким образом, в определенном смысле компонент $u$ вектора состояния $\left(u, v_{1}, v_{2}, v_{3}\right)$ является главным.

Дополним уравнения (4.17) и (4.18) начальными данными

$$
\left.u\right|_{t=0}=u_{0}(x),\left.\quad v_{j}\right|_{t=0}=v_{j 0}(x), \quad j=1,2,3,
$$

и рассмотрим для определенности случай периодических граничных условий:

$$
u(x+L, t)-u(x, t)=d_{j} \cdot\left(v_{j}(x+L, t)-v_{j}(x, t)\right)=0,
$$

где $j=1,2,3, x \in \mathbb{R}^{1}, t>0$. Таким образом, для функции $v_{j}(x, t)$ не ставится каких-либо граничных условий в том случае, когда соответствуюший коэффициент диффузии $d_{j}$ равен нулю, $j=1,2,3$.

Опишем теперь некоторые свойства решений задачи (4.17)-(4.20). Прежде всего, отметим (см. [39]), что параллелепипед

$$
D=\left\{U \equiv\left(u, v_{1}, v_{2}, v_{3}\right): \delta_{2} \leqslant u \leqslant \delta_{1}, 0 \leqslant v_{j} \leqslant 1, j=1,2,3\right\} \subset \mathbb{R}^{4}
$$

является положительно инвариантной областью задачи (4.17)-(4.20). Это означает, что если начальные данные $U_{0}(x)=\left(u_{0}(x), v_{10}(x), v_{20}(x), v_{30}(x)\right)$ принадлежат $D$ для почти всех $x \in[0, L]$, то

$$
U(t) \equiv\left(u(x, t), v_{1}(x, t), v_{2}(x, t), v_{3}(x, t)\right) \in D
$$

для $x \in[0, L]$ и всех $t>0$, для которых решение сушествует.

Пусть $\mathbf{H}_{s} \equiv\left[V_{s}\right]^{4} \equiv\left[H_{\mathrm{per}}^{s}(0, L)\right]^{4}-$ пространство, состоящее из вектор-функщий $U(x) \equiv\left(u, v_{1}, v_{2}, v_{3}\right)$, где $u, v_{j} \in H_{\mathrm{per}}^{s}(0, L), j=1,2,3$. Снабдим его стандартной нормой. Здесь и ниже $V_{s}=H_{\mathrm{per}}^{s}(0, L)$ - подпространства соболевского пространства порядка $s$ на $(0, L)$, отвечаюшие граничным условиям (4.20). Для нормы и скалярного произведения в $V_{0} \equiv L^{2}(0, L)$ используются обозначения $\|\cdot\|$ и $(\cdot, \cdot)$. Пусть

$$
\mathbf{H}_{0}(D)=\left\{U(x) \in \mathbf{H}_{0}: U(x) \in D \text { для почти всех } x \in(0, L)\right\} .
$$

Предположим, что $d_{j}>0$ для всех $j=1,2,3$. Тогда для каждого вектора $U_{0} \in$ $\mathbf{H}_{0}(D) \cap \mathbf{H}_{1}$ задача (4.17)-(4.20) имеет единственное решение $U(t)$, определенное для всех $t$ (см., например, [2], [38]). Это решение лежит в

$$
C\left(\mathbb{R}_{+} ; \mathbf{H}_{0}(D) \cap \mathbf{H}_{1}\right) \cap L_{\mathrm{loc}}^{2}\left(\mathbb{R}_{+} ; \mathbf{H}_{2}\right) .
$$


Если же $d_{1}=d_{2}=d_{3}=0$, то применение обших методов, представленных в [38] (см. также [40]), позволяет показать, что для каждого вектора $U_{0} \in \mathbf{H}_{0}(D) \cap \mathbf{H}_{1}$ задача (4.17)-(4.20) имеет единственное решение в пространстве

$$
C\left(\mathbb{R}_{+} ; \mathbf{H}_{0}(D) \cap \mathbf{H}_{1}\right) \cap L_{\mathrm{loc}}^{2}\left(\mathbb{R}_{+} ; H_{\mathrm{per}}^{2}(0, L) \times\left(H_{\mathrm{per}}^{1}(0, L)\right)^{3}\right) .
$$

Следующее утверждение является непосредственным следствием теоремы 4.3, если переопределить нормы (и соответственно, скалярные произведения) в пространствах $\mathbf{H}_{s}$ с помошью формул

$$
\|U\|_{\mathbf{H}_{s}}^{2}=\left\|\partial_{x}^{s} u\right\|_{L^{2}(0, L)}^{2}+\sum_{j=0}^{3} \theta_{j}^{(s)} \cdot\left\|\partial_{x}^{s} v_{j}\right\|_{L^{2}(0, L)}^{2}, \quad s=0,1,
$$

с подходящим выбором констант $\theta_{j}^{(s)}>0$. Здесь $U=\left(u, v_{1}, v_{2}, v_{3}\right)$. Некоторые детали рассуждений для более сложного случая можно найти ниже в доказательстве теоремы 4.5 .

Tеорема 4.4. Пусть $\mathscr{L}=\left\{l_{j}: j=1, \ldots, N\right\}$ - конечное множество функиионалов на пространстве $V_{1}$. Предположим, что

$$
\varepsilon \mathscr{L} \equiv \varepsilon_{\mathscr{L}}\left(V_{1}, V_{0}\right)<\sqrt{\frac{d_{0}}{d_{0}+K}},
$$

әде

$$
K=\left(\delta_{1}-\delta_{2}\right) \cdot \sum_{j=1}^{3} \frac{\beta_{j}\left(A_{j}+B_{j}\right)}{k_{j}^{*}}
$$

$c \beta_{1}=3 \gamma_{1}, \beta_{2}=\gamma_{1}, \beta_{3}=4 \gamma_{2} u$

$$
\begin{gathered}
A_{j}=\max \left\{\left|\partial_{u} k_{j}(u)\right|: \delta_{2} \leqslant u \leqslant \delta_{1}\right\}, \\
B_{j}=\max \left\{\left|\partial_{u}\left(k_{j} h_{j}\right)(u)\right|: \delta_{2} \leqslant u \leqslant \delta_{1}\right\}, \\
k_{j}^{*}=\min \left\{k_{j}(u): \delta_{2} \leqslant u \leqslant \delta_{1}\right\} .
\end{gathered}
$$

Пусть $P$ - оператор в $\mathbf{H}_{0}$, которьй вектору $U_{0} \in \mathbf{H}_{0}$ ставит в соответствие его первый компонент и. Тогда множество $P^{*} \mathscr{L}$ функиионалов вида $l_{j}^{P}(U)=$ $l_{j}(P U)$, где $l_{j} \in \mathscr{L}, j=1, \ldots, N$, является асимптотически $\left(\mathbf{H}_{1}, \mathbf{H}_{0}, \mathscr{W}\right)$-определяющим множеством для задачи (4.17)-(4.20). Здесь $\mathscr{W}$ либо имеет вид (4.21) при $d_{j}>0$, либо совпадает с (4.22), если $d_{j}=0, j=1,2,3$.

Используя эту теорему и результаты $\S 2$ и $\S 3$, можно, в частности, показать, что множество узлов

$$
\mathscr{L}=\left\{l_{j}: l_{j}(w)=w\left(x_{j}\right), x_{j}=j h, h=\frac{L}{N}, j=1, \ldots, N_{h}\right\}
$$

является асимптотически определяющим, если $N>N_{0} \equiv c_{0} L \cdot \sqrt{1+\frac{K}{d_{0}}}$, где $c_{0}-$ некоторая абсолютная константа. Подобные оценки справедливы для мод и обобщенных локальных объемных средних. Таким образом, для того чтобы асимптотическая 
динамика системы определялась небольшим числом функционалов, приходится требовать малость параметра $N_{0}$. Однако, используя имеющиеся результаты (см. [41]) об аналитичности решений задачи (4.17)-(4.20), можно показать (см. приведенную ниже теорему 4.5), что значения всех компонентов вектора состояния $U=\left(u, v_{1}, v_{2}, v_{3}\right)$ в двух достаточно близких узлах однозначно определяют асимптотическую динамику рассматриваемой системы независимо от величины параметра $N_{0}$. При этом необходимы некоторые условия регулярности коэффициентов уравнений (4.17) и (4.18).

Предположим, что $d_{j}>0$ для всех $j$, а функции $k_{j}(u)$ и $h_{j}(u)$ являются полиномами такими, что $k_{j}(u)>0$ и $0 \leqslant h_{j}(u) \leqslant 1$ при $u \in\left[\delta_{2}, \delta_{1}\right]$. В этом случае (см. [41]) любое решение

$$
U(t)=\left(u(x, t), v_{1}(x, t), v_{2}(x, t), v_{3}(x, t)\right)
$$

обладает следуюшим свойством регулярности Жевре: существует $t_{*}>0$ такое, что

$$
\sum_{l=-\infty}^{\infty}\left(\left|F_{l}(u(t))\right|^{2}+\sum_{j=1}^{3}\left|F_{l}\left(v_{j}(t)\right)\right|^{2}\right) \cdot e^{\tau|l|} \leqslant C
$$

для некоторого $\tau>0$ и всех $t \geqslant t_{*}$. Здесь $F_{l}(w)$ - коэффициенты Фурье функции $w(x)$ :

$$
F_{l}(w)=\frac{1}{L} \cdot \int_{0}^{L} w(x) \cdot \exp \left\{i \frac{2 \pi l}{L} x\right\} d x, \quad l=0, \pm 1, \pm 2, \ldots
$$

В частности, свойство (4.25) означает, что все решения задачи (4.17)-(4.20) становятся вешественно-аналитическими функциями, начиная с некоторого момента времени. Это свойство позволяет доказать следующее утверждение.

TеОРема 4.5. Пусть $d_{j}>0$ для всех $j$, a $k_{j}(u) u h_{j}(u)-$ полиномьи, обладающие свойствами $k_{j}(u)>0 u 0 \leqslant h_{j}(u) \leqslant 1$ при $u \in\left[\delta_{2}, \delta_{1}\right]$. Пусть $x_{1} u x_{2}-$ два узла такие, что $0 \leqslant x_{1}<x_{2} \leqslant L u x_{2}-x_{1}<\sqrt{2 d_{0} / K}$, где K определяется формулой (4.24). Тогда множество функчионалов

$$
\mathscr{L}=\left\{l_{0 n}(U)=u\left(x_{n}\right), l_{j n}(U)=v_{j}\left(x_{n}\right): n=1,2 ; j=1,2,3\right\}
$$

является асимптотически $\left(\mathbf{H}_{1}, \mathbf{H}_{0}, \mathscr{W}\right)$-определяющим множеством для задачи (4.17)-(4.20). Здесь $\mathscr{W}$ имеет вид (4.21).

ДокАЗАТЕльство. Пусть $W=\left(w, \psi_{1}, \psi_{2}, \psi_{3}\right)$ - разность двух решений, лежаших в классе (4.21). Введем обозначения:

$$
\Delta=\left\{x: x_{1} \leqslant x \leqslant x_{2}\right\}, \quad|\Delta|=x_{2}-x_{1} \quad \text { и }\|w\|_{\Delta}^{2}=\int_{x_{1}}^{x_{2}}|w(x)|^{2} d x .
$$

Пусть

$$
m(t, \Delta)=\max _{n=1,2}\left\{\left|w\left(x_{n}, t\right)\right|+\sum_{j=1}^{3}\left|\psi_{j}\left(x_{n}, t\right)\right|\right\}
$$


Обычное интегрирование по частям дает, что

$$
\begin{aligned}
\frac{1}{2} \cdot \frac{d}{d t}\|w\|_{\Delta}^{2}+d_{0}\left\|\partial_{x} w\right\|_{\Delta}^{2}+\gamma_{3}\|w\|_{\Delta}^{2} & \\
& \leqslant \sum_{n=1,2}\left|w\left(x_{n}, t\right) \cdot \partial_{x} w\left(x_{n}, t\right)\right|+\left(\delta_{1}-\delta_{2}\right) \sum_{j=1}^{3} \beta_{j}\left\|\psi_{j}\right\|_{\Delta} \cdot\|w\|_{\Delta}
\end{aligned}
$$

и

$$
\frac{1}{2} \cdot \frac{d}{d t}\left\|\psi_{j}\right\|_{\Delta}^{2}+k_{j}^{*}\left\|\psi_{j}\right\|_{\Delta}^{2} \leqslant \sum_{l=1,2}\left|\psi_{j}\left(x_{l}, t\right) \cdot \partial_{x} \psi_{j}\left(x_{l}, t\right)\right|+\left(A_{j}+B_{j}\right)\left\|\psi_{j}\right\|_{\Delta} \cdot\|w\|_{\Delta} .
$$

Из (4.25) вытекает, что

$$
\sup _{t>t_{*}} \max _{x \in[0, L]}\left\{\left|\partial_{x} w(x, t)\right|+\sum_{j=1}^{3}\left|\partial_{x} \psi_{j}(x, t)\right|\right\}<\infty .
$$

Поэтому для любого $0<\varepsilon<1$ и $\theta_{j}=\left(\delta_{1}-\delta_{2}\right) \beta_{j} \cdot\left(A_{j}+B_{j}\right)^{-1}, j=1,2,3$, имеем, что

$$
\begin{array}{r}
\frac{1}{2} \cdot \frac{d}{d t}\left(\|w(t)\|_{\Delta}^{2}+\sum_{j=1}^{3} \theta_{j}\left\|\psi_{j}\right\|_{\Delta}^{2}\right)+d_{0}\left\|\partial_{x} w\right\|_{\Delta}^{2}+\gamma_{3}\|w\|_{\Delta}^{2}+\varepsilon \sum_{j=1}^{3} k_{j}^{*} \theta_{j}\left\|\psi_{j}\right\|_{\Delta}^{2} \\
\leqslant K \cdot(1-\varepsilon)^{-1} \cdot\|w\|_{\Delta}^{2}+C m(t, \Delta)
\end{array}
$$

при $t>t_{*}$, где $K$ определяется формулой $(4.24), C$ - некоторая положительная константа. Простое вычисление дает, что

$$
\|w\|_{\Delta}^{2} \leqslant(1+\eta) \frac{|\Delta|^{2}}{2} \cdot \int_{x_{1}}^{x_{2}}\left|\partial_{x} w(x)\right|^{2} d x+C_{\eta} \cdot|\Delta| \cdot\left|w\left(x_{1}\right)\right|^{2}
$$

для любого $\eta>0$. Следовательно, если $|\Delta|^{2}<2 d_{0} / K$, то существуют $0<\varepsilon<1$ и $\eta>0$ такие, что

$$
\frac{d}{d t}\left(\|w\|_{\Delta}^{2}+\sum_{j=1}^{3} \theta_{j}\left\|\psi_{j}\right\|_{\Delta}^{2}\right)+\omega \cdot\left(\|w\|_{\Delta}^{2}+\sum_{j=1}^{3} \theta_{j}\left\|\psi_{j}\right\|_{\Delta}^{2}\right) \leqslant C \cdot m(t, \Delta),
$$

где $\omega$ - положительная константа. Отсюда (см. лемму 1.1) заключаем, что из условия $m(t, \Delta) \rightarrow 0, t \rightarrow \infty$, вытекает соотношение

$$
\lim _{t \rightarrow \infty}\left(\|w\|_{\Delta}^{2}+\sum_{j=1}^{3} \theta_{j}\left\|\psi_{j}\right\|_{\Delta}^{2}\right)=0
$$

Используя теперь тот факт, что все решения стремятся к глобальному аттрактору, состоящему из вешественно-аналитических функций, и рассуждая так же, как и в [15], [42], можно завершить доказательство теоремы.

Отметим, что впервые внимание на связь между свойством регулярности Жевре и сушествованием малого числа определяюших узлов было обращено в работе [15], посвяшенной уравнению Гинзбурга-Ландау (см. также [16], [42]). Интересно, что в случае уравнения Курамото-Сивашинского число определяюших узлов оказалось равным четырем [16]. 


\section{$\S 5$. Определяющие функционалы для уравнений второго порядка по времени}

В сепарабельном гильбертовом пространстве $H$ рассмотрим задачу вида

$$
\frac{d^{2} u}{d t^{2}}+\gamma \frac{d u}{d t}+A u+G(u, t)=0, \quad t>0,\left.\quad u\right|_{t=0}=u_{0},\left.\quad \frac{d u}{d t}\right|_{t=0}=u_{1} .
$$

Здесь, как и ранее (см. §4), $A$ - положительный оператор с дискретным спектром, $\gamma-$ положительная константа. Предполагается, что функция $G(u, t)$ является непрерьвным отображением из $D\left(A^{1 / 2}\right) \times \mathbb{R}$ в пространство $H$, обладаюшим при некотором $0 \leqslant \theta<1 / 2$ свойством

$$
\left\|G\left(u_{1}, t\right)-G\left(u_{2}, t\right)\right\| \leqslant M(\rho)\left\|A^{\theta}\left(u_{1}-u_{2}\right)\right\|
$$

для всех $u_{j} \in D\left(A^{1 / 2}\right)$ таких, что $\left\|A^{1 / 2} u_{j}\right\| \leqslant \rho$, где $\rho$ - произвольное положительное число, $M(\rho)>0$ - некоторая константа, $\|\cdot\|=\|\cdot\|_{H}$ и $(\cdot, \cdot)$ - норма и скалярное произведение в $H$. Предположим, что задача (5.1) однозначно разрешима в классе функций

$$
\mathscr{W}=C^{1}([0,+\infty) ; H) \cap C\left([0,+\infty) ; D\left(A^{1 / 2}\right)\right)
$$

и является точечно диссипативной в следуюшем смысле: существует $R>0$ такое, что

$$
\left\|\partial_{t} u(t)\right\|^{2}+\left\|A^{1 / 2} u(t)\right\|^{2} \leqslant R^{2} \text { при } t \geqslant t_{0}(u)
$$

для всех $u(t) \in \mathscr{W}$. Примеры задач вида (5.1), для которых вьполнены приведенные вьше свойства, можно найти, например, в [1] и [2] (см. также [4]). Этому классу задач принадлежит, в частности, нелинейное волновое уравнение вида

$$
\begin{gathered}
\frac{\partial^{2} u}{\partial t^{2}}+\gamma \frac{\partial u}{\partial t}-\Delta u+f(u)=0, \quad x \in \Omega, \quad t>0 \\
\left.u\right|_{\partial \Omega}=0,\left.\quad u\right|_{t=0}=u_{0}(x),\left.\quad \frac{\partial u}{\partial t}\right|_{t=0}=u_{1}(x) .
\end{gathered}
$$

Здесь $\Omega$ - гладкая ограниченная область в $\mathbb{R}^{d}, f(u)$ - подходящая непрерьвно дифференцируемая функция, $\gamma$ - положительньй параметр.

В этом параграфе приведено (вместе с примерами) несколько утверждений о сушествовании определяюших функционалов для задачи (5.1) и ее запаздываюших возмушений. Основную роль здесь играет теорема 1.1. Соответствующая функция $V(u, t)$, хотя и является квадратичной, имеет более сложную структуру по сравнению с параболическим (см. §4) случаем.

Teорема 5.1. Пусть $\mathscr{L}=\left\{l_{j}: j=1, \ldots, N\right\}$ - множество линейных непрерьвных функиионалов на пространстве $V=D\left(A^{1 / 2}\right)$, снабженном нормой $\|\cdot\|_{V}=\left\|A^{1 / 2} \cdot\right\|$. Предположим, ито дефект полноты $\varepsilon=\varepsilon_{\mathscr{L}}(V, H)$ удовлетворяет неравенству

$$
32 \gamma^{-2} M(R)^{2} \varepsilon_{\mathscr{L}}^{2-4 \theta}+\gamma^{2} \varepsilon_{\mathscr{L}}^{2}<8,
$$


где $M(\rho), R$ и $\gamma$ - величиньи, фигурирующие в (5.1), (5.2) и (5.4). Тогда $\mathscr{L}$ является множеством асимптотически $(V, V \times H, \mathscr{W})$-определяющих функиионалов задачи (5.1) в следующем смысле: для любой пары ее решений $u_{1}, u_{2} \in \mathscr{W}$ из условия

$$
\lim _{t \rightarrow \infty} \int_{t}^{t+1}\left|l_{j}\left(u_{1}(\tau)\right)-l_{j}\left(u_{2}(\tau)\right)\right|^{2} d \tau=0 \quad \partial \Omega_{s} \quad j=1, \ldots, N
$$

вьтекает, что

$$
\lim _{t \rightarrow \infty}\left\{\left\|A^{1 / 2}\left(u_{1}(t)-u_{2}(t)\right)\right\|^{2}+\left\|\partial_{t} u_{1}(t)-\partial_{t} u_{2}(t)\right\|^{2}\right\}=0 .
$$

ДокаЗАТЕЛЬСТво. Пусть $u_{1}(t), u_{2}(t) \in \mathscr{W}-$ решения задачи $(5.1)$ и $w(t)=$ $u_{1}(t)-u_{2}(t)$. Используя (5.1) и (5.2), легко обнаружить, что

$$
\frac{1}{2} \frac{d}{d t}\left(\left\|\partial_{t} w(t)\right\|^{2}+\left\|A^{1 / 2} w(t)\right\|^{2}\right)+\frac{3}{4} \gamma\left\|\partial_{t} w(t)\right\|^{2} \leqslant \frac{M(R)^{2}}{\gamma}\left\|A^{\theta} w(t)\right\|^{2}
$$

для достаточно больших $t$. Далее, простая проверка показьвает, что

$$
\begin{aligned}
& \frac{d}{d t}\left(\left(w(t), \partial_{t} w(t)\right)+\frac{\gamma}{2}\|w(t)\|^{2}\right) \\
& \leqslant\left\|\partial_{t} w(t)\right\|^{2}-\left\|A^{1 / 2} w(t)\right\|^{2}+\frac{2 M(R)^{2}}{\gamma^{2}}\left\|A^{\theta} w(t)\right\|^{2}+\frac{\gamma^{2}}{8}\|w(t)\|^{2}
\end{aligned}
$$

для достаточно больших $t$. Рассмотрим теперь функцию

$$
\begin{aligned}
V(t) & \equiv V\left(w(t), \partial_{t} w(t)\right) \\
& =\frac{1}{2}\left(\left\|\partial_{t} w(t)\right\|^{2}+\left\|A^{1 / 2} w(t)\right\|^{2}\right)+\frac{\gamma}{2} \cdot\left(\left(w(t), \partial_{t} w(t)\right)+\frac{\gamma}{2}\|w(t)\|^{2}\right) .
\end{aligned}
$$

Очевидно, что

$$
\frac{1}{4}\left(\left\|\partial_{t} w(t)\right\|^{2}+\left\|A^{1 / 2} w(t)\right\|^{2}\right) \leqslant V(t) \leqslant \frac{3}{4}\left\|\partial_{t} w(t)\right\|^{2}+\frac{1}{2}\left\|A^{1 / 2} w(t)\right\|^{2}+\frac{\gamma^{2}}{2}\|w(t)\|^{2} .
$$

Поэтому из (5.8)-(5.10) вытекает, что для всех $\beta \in(0, \gamma / 3]$ :

$$
\frac{d}{d t} V(t)+\beta V(t) \leqslant-\frac{\gamma-\beta}{2}\left\|A^{1 / 2} w(t)\right\|^{2}+\frac{2 M(R)^{2}}{\gamma}\left\|A^{\theta} w(t)\right\|^{2}+\frac{\gamma^{2}}{16}(\gamma+8 \beta)\|w(t)\|^{2} .
$$

Пользуясь неравенством $\left\|A^{\theta} w\right\| \leqslant\left\|A^{1 / 2} w\right\|^{2 \theta} \cdot\|w\|^{1-2 \theta}$ при $0 \leqslant \theta<1 / 2$ и леммой 2.1, получаем, что

$$
\varepsilon_{\mathscr{L}}\left(D\left(A^{1 / 2}\right), D\left(A^{\theta}\right)\right) \leqslant \varepsilon_{\mathscr{L}}\left(D\left(A^{1 / 2}\right), H\right)^{1-2 \theta} \equiv \varepsilon_{\mathscr{L}}^{1-2 \theta} .
$$

Поэтому с помощью теоремы 2.1 находим, что

$$
\frac{d}{d t} V(t)+\beta V(t) \leqslant-\frac{\omega}{16}\left\|A^{1 / 2} w(t)\right\|^{2}+C_{\mathscr{L}, \delta} \max _{j}\left|l_{j}(w(t))\right|^{2},
$$

где

$$
\omega=8(\gamma-\beta)-32 \gamma^{-1} M(R)^{2} \varepsilon_{\mathscr{L}}^{2-4 \theta}-\gamma^{2}(\gamma+8 \beta) \varepsilon_{\mathscr{L}}^{2}-\delta
$$

с любым достаточно малым $\delta>0$. Используя это соотношение, легко показать, что при условии (5.5) в окрестности нуля существуют $\delta>0$ и $\beta>0$ такие, что $\omega>0$. Поэтому в силу теоремы 1.1 из (5.6) вытекает (5.7). Теорема 5.1 доказана. 
ПРИмеР 5.1. При изучении динамики упругой пологой оболочки в сверхзвуковом потоке газа часто используется следуюшая система уравнений Кармана (см., например, [4], [43], [44] и ссылки, приведенные в этих работах):

$$
\begin{aligned}
& (1-\alpha \cdot \Delta) \partial_{t}^{2} u+\gamma(1-\alpha \cdot \Delta) \partial_{t} u+\Delta^{2} u \\
& \quad-[u+f, v+\psi]+\beta \partial_{x_{1}} u=p(x), \quad x \in \Omega, \quad t>0, \\
& \left.u\right|_{\partial \Omega}=\left.\frac{\partial u}{\partial n}\right|_{\partial \Omega}=0,\left.\quad u\right|_{t=0}=u_{0}(x),\left.\quad \partial_{t} u\right|_{t=0}=u_{1}(x) .
\end{aligned}
$$

Величина $v=v(u)$ определяется как решение задачи

$$
\Delta^{2} v+[u+2 f, u]=0,\left.\quad v\right|_{\partial \Omega}=\left.\frac{\partial v}{\partial n}\right|_{\partial \Omega}=0
$$

Здесь $\Omega$ - гладкая ограниченная область в $\mathbb{R}^{2}, \Delta$ - оператор Лапласа и

$$
[u, v]=\partial_{x_{1}}^{2} u \cdot \partial_{x_{2}}^{2} v+\partial_{x_{2}}^{2} u \cdot \partial_{x_{1}}^{2} v-2 \cdot \partial_{x_{1} x_{2}}^{2} u \cdot \partial_{x_{1} x_{2}}^{2} v
$$

Неизвестная функщия $u=u(x, t)$ описывает поперечное отклонение оболочки в точке $x$ в момент $t ; \psi(x) \in H^{4}(\Omega), f(x) \in H^{2}(\Omega)$ и $p(x) \in L^{2}(\Omega)$ - заданные функции, определяемые механическими нагрузками и исходной формой оболочки. Параметр $\alpha>0$ учитывает инерцию вращения элементов оболочки. Число $\beta>0$ определяется скоростью набегающего вдоль оси $x_{1}$ потока газа. Величина $\gamma>0$ описьвает сопротивление среды. Задача (5.13)-(5.15) может быть записана как уравнение вида (5.1) в пространстве $H_{0}^{1}(\Omega)$. Все условия, предъявляемые вьше к задаче $(5.1)$, вьполняются (см. [43]) и для (5.13)-(5.15). При этом $\theta$ - любое число из интервала $(1 / 4,1 / 2)$, а величина $M(\rho)$ имеет вид

$$
M(\rho)=c_{0}\left(\beta+\|f\|_{H^{2}(\Omega)}^{2}+\|\psi\|_{H^{4}(\Omega)}+\rho^{2}\right)
$$

где $c_{0}$ - некоторая константа, зависяшая от размеров области $\Omega$. Решения рассматриваются в классе

$$
\mathscr{W}=C^{1}\left([0,+\infty) ; H_{0}^{1}(\Omega)\right) \cap C\left([0,+\infty) ; H_{0}^{2}(\Omega)\right) .
$$

Применение теоремы 5.1 дает следуюшее утверждение.

Теорема 5.2. Пусть $\mathscr{L}=\left\{l_{j}: j=1, \ldots, N\right\}$ - множество линейных непрерывных функиионалов на пространстве $H_{0}^{2}(\Omega)$. Тогда существует число $\varepsilon_{0}>0$, зависящее от параметров уравнения (5.13), такое, что из условия $\varepsilon \mathscr{L}\left(H_{0}^{2}(\Omega), H_{0}^{1}(\Omega)\right)<\varepsilon_{0}$ вытекает, что $\mathscr{L}$ является множеством асимптотически $\left(H_{0}^{2}(\Omega), H_{0}^{2}(\Omega) \times H_{0}^{1}(\Omega), \mathscr{W}\right)$-определяюших функчионалов задачи (5.13)-(5.15), т.е. для любых двух решений $u_{1}, u_{2} \in \mathscr{W}$ из условия (5.6) вьтекает, что

$$
\lim _{t \rightarrow \infty}\left\{\left\|u_{1}(t)-u_{2}(t)\right\|_{H^{2}(\Omega)}^{2}+\left\|\partial_{t} u_{1}(t)-\partial_{t} u_{2}(t)\right\|_{H^{1}(\Omega)}^{2}\right\}=0
$$


Аналогичное утверждение может быть установлено и для задачи (5.13)-(5.15) в случае, когда $\alpha=0$ (см. [18] и [21]). Соответствующая функция $V(t)$ (см. (5.10)) имеет более сложный вид. Отметим также, что результаты $\S 3$ гарантируют для задачи (5.13)-(5.15) существование конечных наборов асимптотически определяюших узлов и локальных объемных средних. Однако получить эффективные оценки величины $\varepsilon_{0}$, фигурируюшей в теореме 5.2 , а также соответствующего числа $N$ определяюших функционалов через параметры задачи удается лишь в нескольких частных случаях. Это связано с тем, что имеюшееся доказательство (см. [43]) диссипативности задачи (5.13)-(5.15) не является конструктивным.

Другой интересный класс примеров возникает, если рассмотреть запаздьваюшие возмушения задачи (5.1):

$$
\begin{gathered}
\frac{d^{2} u}{d t^{2}}+\gamma \frac{d u}{d t}+A u+G(u, t)=Q\left(u_{t}, t\right), \quad t>0, \\
\left.u\right|_{t=+0}=u_{0},\left.\quad \frac{d u}{d t}\right|_{t=+0}=u_{1},\left.\quad u\right|_{t \in(-r, 0)}=\phi(t) \in L^{2}(-r, 0 ; H) .
\end{gathered}
$$

Здесь, как обычно (см. [45]), использовано обозначение $u_{t}$ для элемента из $L^{2}(-r, 0 ; H)$, определяемого равенством $u_{t}(s)=u(t+s), s \in(-r, 0)$. Параметр $r>0$ имеет смысл величины запаздывания. Предполагается, что $G(u, t)$ обладает свойством $(5.2)$, а $Q(\cdot, t)$ - непрерьвное линейное отображение $L^{2}\left(-r, 0 ; D\left(A^{1 / 2}\right)\right)$ в $H$, обладающее для некоторого $\theta^{*} \in[0,1 / 2)$ свойством

$$
\|Q(u, t)\|^{2} \leqslant q_{0} \int_{t-r}^{t}\left\|A^{\theta^{*}} u(\tau)\right\|^{2} d \tau,
$$

где $q_{0}$ - некоторая константа. Предположим также, что задача (5.16)-(5.17) однозначно разрешима в классе функций

$$
\mathscr{W}=C^{1}([0,+\infty) ; H) \cap C\left([0,+\infty) ; D\left(A^{1 / 2}\right)\right) \cap L_{\mathrm{loc}}^{2}\left([-r,+\infty) ; D\left(A^{1 / 2}\right)\right)
$$

и является точечно диссипативной, т.е. сушествует $R>0$ такое, что вьполнено (5.4) для всех $u(t) \in \mathscr{W}$. Отметим, что все эти свойства вьполняются для ситуации, рассмотренной в приведенном ниже примере 5.2. Имеет место следуюшее утверждение.

Tеорема 5.3. Пусть $\mathscr{L}=\left\{l_{j}: j=1, \ldots, N\right\}$ - множество линейных непрерывных функционалов на пространстве $V=D\left(A^{1 / 2}\right)$, снабженном нормой $\|\cdot\|_{V}=\left\|A^{1 / 2} \cdot\right\|$. Предположим, что дефект полноты $\varepsilon=\varepsilon \mathscr{L}(V, H)$ удовлетворяет неравенствам

$$
16 \gamma^{-2} M(R)^{2} \varepsilon_{\mathscr{L}}^{2-4 \theta}+\gamma^{2} \varepsilon_{\mathscr{L}}^{2}<2, \quad 12 r q_{0} \gamma^{-2} \varepsilon_{\mathscr{L}}^{2-4 \theta^{*}}<1,
$$

где $M(\rho), R, \gamma, \theta, \theta^{*}, r, q_{0}$ - величиньи, фигурирующие в (5.2), (5.4) и (5.16)-(5.18). Тогда $\mathscr{L}$ является множеством асимптотически $(V, V \times H, \mathscr{W})$-определяющих функиионалов задачи (5.16)-(5.17), т.е. из (5.6) вытекает (5.7). 
ДокАЗАТЕльство. Пусть $u_{1}(t), u_{2}(t) \in \mathscr{W}$ - решения задачи $(5.16)-(5.17)$ и $w(t)=$ $u_{1}(t)-u_{2}(t)$. Рассмотрим функцию $V(t)$, определяемую равенством (5.10). Так же, как и при доказательстве теоремы 5.1, легко проверить (см. (5.8) и (5.9)), что

$$
\begin{aligned}
\frac{d}{d t} V(t) \leqslant-\frac{\gamma}{8}\left\|\partial_{t} w(t)\right\|^{2} & -\frac{\gamma}{2}\left\|A^{1 / 2} w(t)\right\|^{2} \\
& +\frac{2 M(R)^{2}}{\gamma}\left\|A^{\theta} w(t)\right\|^{2}+\frac{\gamma^{3}}{8}\|w(t)\|^{2}+\frac{3}{\gamma}\left\|Q\left(w_{t}, t\right)\right\|^{2}
\end{aligned}
$$

для достаточно больших $t>0$. Рассмотрим теперь функцию

$$
W(t)=V(t)+\mu \int_{0}^{r} d s \int_{t-s}^{t}\left\|A^{1 / 2} w(\tau)\right\|^{2} d \tau .
$$

Параметр $\mu>0$ будет выбран ниже. Из (5.11) вытекает, что

$$
W(t) \leqslant \frac{3}{4}\left\|\partial_{t} w(t)\right\|^{2}+\frac{1}{2}\left\|A^{1 / 2} w(t)\right\|^{2}+\frac{\gamma^{2}}{2}\|w(t)\|^{2}+\mu r \int_{t-r}^{t}\left\|A^{1 / 2} w(\tau)\right\|^{2} d \tau
$$

Кроме того, очевидно, что

$$
\frac{d}{d t} W(t)=\frac{d}{d t} V(t)+\mu r\left\|A^{1 / 2} w(t)\right\|^{2}-\mu \int_{t-r}^{t}\left\|A^{1 / 2} w(\tau)\right\|^{2} d \tau .
$$

Поэтому, используя (5.18), из (5.21)-(5.24) для любого $\beta>0$ получаем, что

$$
\begin{aligned}
\frac{d}{d t} W(t)+\beta W(t) \leqslant & -\left(\frac{\gamma}{8}-\frac{3}{4} \beta\right)\left\|\partial_{t} w(t)\right\|^{2}-\left(\frac{\gamma}{2}-\frac{\beta}{2}-\mu r\right)\left\|A^{1 / 2} w(t)\right\|^{2} \\
& +\frac{2 M(R)^{2}}{\gamma}\left\|A^{\theta} w(t)\right\|^{2}+\frac{\gamma^{2}}{8}(\gamma+4 \beta)\|w(t)\|^{2} \\
& +\int_{t-r}^{t}\left(-\mu(1-\beta r)\left\|A^{1 / 2} w(\tau)\right\|^{2}+\frac{3 q_{0}}{\gamma}\left\|A^{\theta^{*}} w(\tau)\right\|^{2}\right) d \tau .
\end{aligned}
$$

Следовательно, как и в теореме 5.1, с помошю (5.12) и свойств дефекта полноты (см. теорему 2.1) находим, что

$$
\begin{aligned}
\frac{d}{d t} W(t)+\beta W(t) & \leqslant-\frac{1}{8}(\gamma-6 \beta)\left\|\partial_{t} w(t)\right\|^{2}-\omega_{1}\left\|A^{1 / 2} w(t)\right\|^{2}-\omega_{2} \int_{t-r}^{t}\left\|A^{1 / 2} w(\tau)\right\|^{2} d \tau \\
& \leqslant C_{\mathscr{L}, \delta}\left(\max _{j}\left|l_{j}(w(t))\right|^{2}+\int_{t-r}^{t} \max _{j}\left|l_{j}(w(\tau))\right|^{2} d \tau\right),
\end{aligned}
$$

где

$$
\begin{aligned}
& \omega_{1}=\left(\frac{\gamma-\beta}{2}-\mu r\right)-\frac{2 M(R)^{2}}{\gamma}(1+\delta) \varepsilon_{\mathscr{L}}^{2-4 \theta}-\frac{\gamma^{2}}{8}(\gamma+4 \beta)(1+\delta) \varepsilon_{\mathscr{L}}^{2}, \\
& \omega_{2}=\mu(1-\beta r)-\frac{3 q_{0}}{\gamma}(1+\delta) \varepsilon_{\mathscr{L}}^{2-4 \theta^{*}}
\end{aligned}
$$

с произвольньми $\delta>0$ и $\beta>0$. Выбирая $\mu=\gamma /(4 r)$, из условий (5.20) получаем, что сушествуют $\delta>0$ и $0<\beta \leqslant \gamma / 6$ такие, что

$$
\frac{d}{d t} W(t)+\beta W(t) \leqslant C_{\mathscr{L}, \delta}\left(\max _{j}\left|l_{j}(w(t))\right|^{2}+\int_{t-r}^{t} \max _{j}\left|l_{j}(w(\tau))\right|^{2} d \tau\right) .
$$

Поэтому теорема 1.1 дает, что из (5.6) вытекает (5.7). Теорема 5.3 доказана. 
ПримеР 5.2. Вернемся к задаче (5.13)-(5.15). Если оболочка находится в потенциальном линеаризованном потоке газа, то аэродинамическое давление потока можно учесть (см. [46], [47] и приведенные там ссылки), полагая в правой части (5.13)

$$
p(x, t)=p_{0}(x)-\frac{\nu}{2 \pi U} q(u ; x, t)
$$

где $p_{0}(x) \in L^{2}(\Omega)$, параметр $\nu>0$ характеризует интенсивность взаимодействия между потоком и оболочкой, величина $U>0(U \neq 1)$ является скоростью невозмущенного потока,

$$
q(u, x, t)=\int_{0}^{r} d s \int_{0}^{2 \pi} d \xi\left[M_{\xi}^{2} \widehat{u}\right]\left(x_{1}-(U+\sin \xi) s, x_{2}-s \cos \xi, t-s\right) .
$$

Здесь $\widehat{u}$ - продолжение функции $u(x, t)$ нулем вне $\Omega$,

$$
M_{\xi}=\sin \xi \cdot \partial_{x_{1}}+\cos \xi \cdot \partial_{x_{2}}
$$

и $r$ - некоторый параметр, зависяший от $U$ и размеров области $\Omega$. Запаздьваюший характер задачи требует добавления к (5.14) начального условия вида

$$
\left.u\right|_{t \in(-r, 0)}=\varphi(x, t)
$$

Из результатов, полученных в [46], [47], вытекает, что задача (5.13)-(5.15), (5.25), $(5.26)$, записанная в пространстве $H_{0}^{1}(\Omega)$, удовлетворяет всем условиям, необходимым для применения теоремы 5.3. Оценка (5.18) вытекает из неравенства (см. [46], [47], а также [48]):

$$
\|q(u, t)\|_{H^{s}(\Omega)}^{2} \leqslant C \int_{t-r}^{t}\|u(\tau)\|_{H^{2+s}(\Omega)}^{2} d \tau \text { для } s=0,-1 .
$$

Таким образом, для системы (5.13)-(5.15), (5.25), (5.26) справедливы заключения теоремы 5.2 с $\theta^{*}=0$. Получить оценки для дефекта полноты в удобной для приложений форме здесь не удается по тем же причинам, что и в примере 5.1.

\section{§ 6. О граничных определяющих функционалах}

Тот факт (см. §4), что в некоторых случаях определяюшие функционалы можно задавать на некотором вспомогательном пространстве, допускает следующую, интересную на наш взгляд, интерпретацию, приводящую к понятию граничных определяющих функционалов. Ниже эта интерпретация разъясняется на примере двух задач.

ПРимеР 6.1. Пусть в гладкой ограниченной области $\Omega \subset \mathbb{R}^{d}$ задано параболическое уравнение с нелинейньм граничным условием:

$$
\left\{\begin{array}{l}
\frac{\partial u}{\partial t}=\nu \Delta u-f(u), \quad x \in \Omega, \quad t>0 \\
\left.\left(\frac{\partial u}{\partial n}+h(u)\right)\right|_{\partial \Omega}=0,\left.\quad u\right|_{t=0}=u_{0}(x)
\end{array}\right.
$$


Здесь $n$ - внешняя нормаль к $\partial \Omega, \nu$ - положительный параметр, а $f(z)$ и $h(z)$ - непрерьвно дифференцируемые функции на $\mathbb{R}^{1}$ такие, что

$$
f^{\prime}(z) \geqslant-\alpha, \quad h^{\prime}(z) \geqslant-\beta, \quad z \in \mathbb{R}^{1}
$$

где $\alpha, \beta \geqslant 0$ - некоторые константы. Рассмотрим множество классических решений (об условиях их существования см., например, в [49]):

$$
\mathscr{W}=C^{2,1}\left(\Omega \times \mathbb{R}_{+}\right) \cap C^{1,0}\left(\overline{\Omega \times \mathbb{R}_{+}}\right) .
$$

Здесь $C^{2,1}\left(\Omega \times \mathbb{R}_{+}\right)$- множество функций $u(x, t)$ на $\Omega \times \mathbb{R}_{+}$, два раза непрерьвно дифференщируемых по $x$ и непрерывно дифференцируемых по $t$. Аналогичньй смысл имеет обозначение $C^{1,0}\left(\overline{\Omega \times \mathbb{R}_{+}}\right)$, черта означает замыкание множества.

Пусть $u_{1}(x, t)$ и $u_{2}(x, t)$ - два решения задачи $(6.1)$, лежашие в классе $\mathscr{W}$. Рассмотрим их разность $u(t)=u_{1}(t)-u_{2}(t)$. Тогда из (6.1) очевидно вытекает, что

$$
\begin{aligned}
\frac{1}{2} \frac{d}{d t}\|u(t)\|_{L^{2}(\Omega)}^{2}+\nu\|\nabla u(t)\|_{L^{2}(\Omega)}^{2}+\left(f\left(u_{1}(t)\right)\right. & \left.-f\left(u_{2}(t)\right), u(t)\right)_{L^{2}(\Omega)} \\
& =-\nu \int_{\partial \Omega} u(t)\left(h\left(u_{1}(t)\right)-h\left(u_{2}(t)\right)\right) d \sigma .
\end{aligned}
$$

Пользуясь (6.2), отсюда получаем, что

$$
\frac{1}{2} \frac{d}{d t}\|u(t)\|_{L^{2}(\Omega)}^{2}+\nu\|\nabla u(t)\|_{L^{2}(\Omega)}^{2}-\alpha\|u(t)\|_{L^{2}(\Omega)}^{2} \leqslant \nu \beta\|u(t)\|_{L^{2}(\partial \Omega)}^{2} .
$$

Можно показать, что сушествуют константы $a_{1}$ и $a_{2}$, зависяшие лишь от области $\Omega$, такие, что

$$
\|u\|_{L^{2}(\Omega)}^{2} \leqslant a_{1}\left(\|u\|_{L^{2}(\partial \Omega)}^{2}+\|\nabla u\|_{L^{2}(\Omega)}^{2}\right)
$$

и

$$
\|u\|_{H^{1 / 2}(\partial \Omega)}^{2} \leqslant a_{2}\left(\|u\|_{L^{2}(\Omega)}^{2}+\|\nabla u\|_{L^{2}(\Omega)}^{2}\right)
$$

Здесь $H^{s}(\partial \Omega)$ - соболевское пространство порядка $s$ на гранище области $\Omega$. Соотношения (6.4)-(6.6) позволяют получить следуюшее утверждение, являющееся фактически некоторой конкретизацией теоремы 4.3 .

Теорема 6.1. Пусть $\mathscr{L}=\left\{l_{j}: j=1, \ldots, N\right\}-$ множсество функиионалов на пространстве $H^{1 / 2}(\partial \Omega)$. Предположим, что $\alpha \cdot a_{1}<\nu u$

$$
\varepsilon \mathscr{L} \equiv \varepsilon \mathscr{L}\left(H^{1 / 2}(\partial \Omega), L^{2}(\partial \Omega)\right)<\sqrt{\left(1-\alpha \nu^{-1} a_{1}\right) a_{3}},
$$

где $a_{3}^{-1}=\left(1+a_{1}\right) a_{2}(1+\beta), \quad$ к константы $\nu, \alpha, \beta, a_{1}, a_{2}$ определень $\boldsymbol{в}$ соотношениях (6.1), (6.2), (6.5), (6.6). Тогда $\mathscr{L}$ является асимптотически $\left(H^{1 / 2}(\partial \Omega), L^{2}(\Omega), \mathscr{W}\right)$-определяющим множеством для задачи (6.1) в классе классических решений (6.3). 
ДокАЗАТЕЛЬСТВо. Пусть $u(t)=u_{1}(t)-u_{2}(t)$, где $u_{j}(t) \in \mathscr{W}-$ решения задачи (6.1). Из теоремы 2.1 вытекает, что

$$
\|u\|_{L^{2}(\partial \Omega)}^{2} \leqslant C_{\mathscr{L}, \eta} \max _{j}\left|l_{j}\left(\left.u\right|_{\partial \Omega}\right)\right|^{2}+(1+\eta) \varepsilon_{\mathscr{L}}^{2}\|u\|_{H^{1 / 2}(\partial \Omega)}^{2}
$$

для любого $\eta>0$. Поэтому из (6.5) и (6.6) вытекает, что

$$
\|u\|_{L^{2}(\partial \Omega)}^{2} \leqslant C \max _{j}\left|l_{j}\left(\left.u\right|_{\partial \Omega}\right)\right|^{2}+\left(1+a_{1}\right) a_{2}(1+\eta) \varepsilon_{\mathscr{L}}^{2}\left(\|u\|_{L^{2}(\partial \Omega)}^{2}+\|\nabla u\|_{L^{2}(\Omega)}^{2}\right) .
$$

Следовательно, (6.4) дает, что

$$
\begin{aligned}
\frac{1}{2} \frac{d}{d t}\|u\|_{L^{2}(\Omega)}^{2} & +\nu\left[\|u\|_{L^{2}(\partial \Omega)}^{2}+\|\nabla u\|_{L^{2}(\Omega)}^{2}\right]-\alpha\|u(t)\|_{L^{2}(\Omega)}^{2} \\
& \leqslant \nu(1+\eta) a_{3}^{-1} \cdot \varepsilon_{\mathscr{L}}^{2}\left(\|u\|_{L^{2}(\partial \Omega)}^{2}+\|\nabla u\|_{L^{2}(\Omega)}^{2}\right)+C \max _{j}\left|l_{j}\left(\left.u\right|_{\partial \Omega}\right)\right|^{2} .
\end{aligned}
$$

Используя еще раз оценку (6.5), имеем, что

$$
\frac{d}{d t}\|u\|_{L^{2}(\Omega)}^{2}+\frac{2 \nu}{a_{1}}\left\{1-\frac{1+\eta}{a_{3}} \varepsilon_{\mathscr{L}}^{2}-\alpha \frac{a_{1}}{\nu}\right\}\|u\|_{L^{2}(\Omega)}^{2} \leqslant C \max _{j}\left|l_{j}\left(\left.u\right|_{\partial \Omega}\right)\right|^{2},
$$

при условии, что $1-(1+\eta) a_{3}^{-1} \varepsilon_{\mathscr{L}}^{2}-\alpha a_{1} \nu^{-1}>0$. Очевидно, что это условие при некотором $\eta>0$ вытекает из (6.7). Поэтому неравенство (6.9) позволяет завершить доказательство теоремы.

Таким образом, асимптотическая динамика решений задачи (6.1) однозначно определяется поведением их следов на границе области. В частности, аналог теоремы 3.1 для гладких поверхностей позволяет утверждать, что задача (6.1) обладает конечными определяюшими наборами граничных локальных поверхностных средних.

ПримеР 6.2. Утверждение, аналогичное теореме 6.1, может быть установлено и для нелинейного волнового уравнения вида

$$
\left\{\begin{array}{l}
\frac{\partial^{2} u}{\partial t^{2}}+\gamma \frac{\partial u}{\partial t}=\nu \Delta u-f(u), \quad x \in \Omega \subset \mathbb{R}^{d}, \quad t>0 \\
\left.\nu \frac{\partial u}{\partial n}\right|_{\Gamma}=-\left.\delta \frac{\partial u}{\partial t}\right|_{\Gamma}-h\left(\left.u\right|_{\Gamma}\right),\left.\quad u\right|_{\partial \Omega \backslash \Gamma}=0 \\
\left.u\right|_{t=0}=u_{0}(x),\left.\quad \frac{\partial u}{\partial t}\right|_{t=0}=u_{1}(x) .
\end{array}\right.
$$

Здесь $n$ - внешняя нормаль к $\partial \Omega, \nu, \gamma, \delta$ - положительные параметры, $\Gamma$ - некоторое гладкое открытое подмножество на границе области $\Omega$, случай $\Gamma=\partial \Omega$ не исключается. Предполагается, что $f(z)$ и $h(z)$ - непрерьвно дифференцируемые функции с ограниченными производными:

$$
\left|f^{\prime}(z)\right| \leqslant \alpha, \quad\left|h^{\prime}(z)\right| \leqslant \beta, \quad z \in \mathbb{R}^{1},
$$

где $\alpha>0, \beta>0$ - некоторые константы. Можно показать [50], что задача (6.10) однозначно разрешима в классе функций

$$
\mathscr{W}=C\left(\mathbb{R}_{+} ; H_{\Gamma}^{1}(\Omega)\right) \cap C^{1}\left(\mathbb{R}_{+} ; L^{2}(\Omega)\right),
$$


где $H_{\Gamma}^{1}(\Omega)=\left\{v \in H^{1}(\Omega):\left.v\right|_{\partial \Omega \backslash \Gamma}=0\right\}$. При этом

$$
\frac{\partial u}{\partial t} \in L^{2}\left(\Gamma \times \mathbb{R}_{+}\right), \quad \frac{\partial u}{\partial n} \in L^{2}\left(\Gamma \times \mathbb{R}_{+}\right)
$$

в смысле обобщенных функщий. Пусть (ср. (6.5), (6.6)) $a_{1}=a_{1}(\Gamma, \Omega)$ и $a_{2}=a_{2}(\Gamma, \Omega)$ - положительные константы такие, что

$$
\|u\|_{L^{2}(\Omega)}^{2} \leqslant a_{1}\left(\|u\|_{L^{2}(\Gamma)}^{2}+\|\nabla u\|_{L^{2}(\Omega)}^{2}\right)
$$

и

$$
\|u\|_{H^{1 / 2}(\Gamma)}^{2} \leqslant a_{2}\left(\|u\|_{L^{2}(\Omega)}^{2}+\|\nabla u\|_{L^{2}(\Omega)}^{2}\right) .
$$

Имеет место следующее утверждение.

Tеорема 6.2. Пусть $\mathscr{L}=\left\{l_{j}: j=1, \ldots, N\right\}$ - множество функиионалов на пространстве $H^{1 / 2}(\Gamma)$. Предположим, ито $\alpha \cdot a_{1}\left(1+2 \alpha \gamma^{-2}\right)<\nu$ и дефект полноты $\varepsilon_{\mathscr{L}} \equiv \varepsilon_{\mathscr{L}}\left(H^{1 / 2}(\Gamma), L^{2}(\Gamma)\right)$ удовлетворяет неравенству

$$
\left(1+a_{1}\right) a_{2} \cdot \varepsilon_{\mathscr{L}}^{2}<2 \gamma\left(\nu-\alpha \cdot a_{1} \frac{2 \alpha+\gamma^{2}}{\gamma^{2}}\right) \cdot\left[\beta^{2} \delta^{-1}+2 \gamma(\nu+\beta)\right]^{-1},
$$

где константы $\nu, \alpha, \beta, \gamma, \delta, a_{1}, a_{2}$ определень в соотношениях (6.10)-(6.13). Тогда $\mathscr{L}$ является асимптотически $\left(H^{1 / 2}(\Gamma),\left(H^{1} \times L^{2}\right)(\Omega), \mathscr{W}\right)$-определяющим множеством для задачи (6.10) в следующем смысле: для любой пары ее решений $u_{1}, u_{2} \in \mathscr{W}$ из условия

$$
\lim _{t \rightarrow \infty} \int_{t}^{t+1}\left|l_{j}\left(\left.u_{1}(\tau)\right|_{\Gamma}\right)-l_{j}\left(\left.u_{2}(\tau)\right|_{\Gamma}\right)\right|^{2} d \tau=0 \quad \partial \Omega_{s} \quad j=1, \ldots, N
$$

витекает, что

$$
\lim _{t \rightarrow \infty}\left\{\left\|u_{1}(t)-u_{2}(t)\right\|_{H^{1}(\Omega)}^{2}+\left\|\partial_{t} u_{1}(t)-\partial_{t} u_{2}(t)\right\|_{L^{2}(\Omega)}^{2}\right\}=0 .
$$

ДокАЗАТЕЛЬСТво. Пусть $u(t)=u_{1}(t)-u_{2}(t)$, где $u_{j}(t) \in \mathscr{W}-$ решения задачи (6.10). Тогда $u(t)$ является решением линейной задачи вида:

$$
\begin{cases}\frac{\partial^{2} u}{\partial t^{2}}+\gamma \frac{\partial u}{\partial t}-\nu \Delta u=f(t) \equiv f\left(u_{2}(t)\right)-f\left(u_{1}(t)\right), & x \in \Omega, t>0, \\ \left.\nu \frac{\partial u}{\partial n}\right|_{\Gamma}+\left.\delta \frac{\partial u}{\partial t}\right|_{\Gamma}=h(t) \equiv h\left(\left.u_{2}(t)\right|_{\Gamma}\right)-h\left(\left.u_{1}(t)\right|_{\Gamma}\right), & \left.u\right|_{\partial \Omega \backslash \Gamma}=0\end{cases}
$$

с некоторыми начальньми условиями. Поэтому предложение 2.1 из [50] для функции

$$
E(t)=\frac{1}{2}\left(\left\|\partial_{t} u(t)\right\|_{L^{2}(\Omega)}^{2}+\nu\|\nabla u(t)\|_{L^{2}(\Omega)}^{2}\right)
$$


дает, что

$$
\begin{aligned}
E(t)+\int_{0}^{t}\left(\gamma\left\|\partial_{t} u(\tau)\right\|_{L^{2}(\Omega)}^{2}\right. & \left.+\delta\left\|\partial_{t} u(\tau)\right\|_{L^{2}(\Gamma)}^{2}\right) d \tau \\
& =E(0)+\int_{0}^{t}\left(\left(\partial_{t} u(\tau), f(\tau)\right)_{L^{2}(\Omega)}+\left(\partial_{t} u(\tau), h(\tau)\right)_{L^{2}(\Gamma)}\right) d \tau
\end{aligned}
$$

Переписьвая это соотношение в дифференциальной форме, с помошью (6.11) легко обнаружить, что

$$
\frac{d}{d t} E(t)+\frac{3}{4} \gamma\left\|\partial_{t} u(t)\right\|_{L^{2}(\Omega)}^{2} \leqslant \frac{\alpha^{2}}{\gamma}\|u(t)\|_{L^{2}(\Omega)}^{2}+\frac{\beta^{2}}{4 \delta}\|u(t)\|_{L^{2}(\Gamma)}^{2} .
$$

Рассмотрим функцию

$$
\Phi(t)=\left(u(t), \partial_{t} u(t)\right)_{L^{2}(\Omega)}+\frac{\gamma}{2}\|u(t)\|_{L^{2}(\Omega)}^{2}+\frac{\delta}{2}\|u(t)\|_{L^{2}(\Gamma)}^{2} .
$$

Используя некоторые регуляризационные процедуры и результаты из [50], можно показать, что

$$
\frac{d}{d t} \Phi(t) \leqslant\left\|\partial_{t} u(t)\right\|_{L^{2}(\Omega)}^{2}-\nu\|\nabla u(t)\|_{L^{2}(\Omega)}^{2}+\alpha\|u(t)\|_{L^{2}(\Omega)}^{2}+\beta\|u(t)\|_{L^{2}(\Gamma)}^{2}
$$

Очевидно также, что

$$
-\frac{1}{2 \gamma}\left\|\partial_{t} u(t)\right\|_{L^{2}(\Omega)}^{2} \leqslant \Phi(t) \leqslant \frac{1}{2 \gamma}\left\|\partial_{t} u(t)\right\|_{L^{2}(\Omega)}^{2}+\gamma\|u(t)\|_{L^{2}(\Omega)}^{2}+\frac{\delta}{2}\|u(t)\|_{L^{2}(\Gamma)}^{2} .
$$

Поэтому функщия $V(t)=E(t)+\frac{\gamma}{2} \Phi(t)$ обладает свойствами

$$
\begin{aligned}
\frac{d}{d t} V(t) \leqslant-\frac{\gamma}{4}\left\|\partial_{t} u(t)\right\|_{L^{2}(\Omega)}^{2}-\frac{\gamma \nu}{2}\|\nabla u(t)\|_{L^{2}(\Omega)}^{2} & \\
& +\frac{\alpha}{2}\left(2 \alpha \gamma^{-1}+\gamma\right)\|u(t)\|_{L^{2}(\Omega)}^{2}+\frac{\beta}{4}\left(\beta \delta^{-1}+2 \gamma\right)\|u(t)\|_{L^{2}(\Gamma)}^{2}
\end{aligned}
$$

и

$$
\frac{1}{2} E(t) \leqslant V(t) \leqslant \frac{3}{4}\left\|\partial_{t} u(t)\right\|_{L^{2}(\Omega)}^{2}+\frac{\nu}{2}\|\nabla u(t)\|_{L^{2}(\Omega)}^{2}+\frac{\gamma^{2}}{2}\|u(t)\|_{L^{2}(\Omega)}^{2}+\frac{\delta \gamma}{4}\|u(t)\|_{L^{2}(\Gamma)}^{2} .
$$

Отсюда вытекает, что для любого $\omega>0$ справедливо соотношение

$$
\begin{aligned}
& \frac{d}{d t} V(t)+\omega V(t) \leqslant-\frac{1}{4}(\gamma-3 \omega)\left\|\partial_{t} u(t)\right\|_{L^{2}(\Omega)}^{2}-\frac{\nu}{2}(\gamma-\omega)\|\nabla u(t)\|_{L^{2}(\Omega)}^{2} \\
& \quad+\frac{1}{2}\left(2 \alpha^{2} \gamma^{-1}+\alpha \gamma+\omega \gamma^{2}\right)\|u(t)\|_{L^{2}(\Omega)}^{2}+\frac{1}{4}\left(\beta^{2} \delta^{-1}+2 \beta \gamma+\delta \gamma \omega\right)\|u(t)\|_{L^{2}(\Gamma)}^{2} .
\end{aligned}
$$

Используя (6.8) с Г вместо $\partial \Omega$, а также соотношения (6.12) и (6.13), как и при доказательстве теоремы 6.1 , находим, что при $\omega \leqslant \gamma / 3$ имеет место неравенство

$$
\frac{d}{d t} V(t)+\omega V(t) \leqslant C \max _{j}\left|l_{j}\left(\left.u\right|_{\Gamma}\right)\right|^{2}-b\left(\|u(t)\|_{L^{2}(\Gamma)}^{2}+\|\nabla u(t)\|_{L^{2}(\Omega)}^{2}\right),
$$


где

$$
\begin{aligned}
b=\frac{\nu}{2}(\gamma-\omega)-\frac{a_{1}}{2}( & \left.2 \alpha^{2} \gamma^{-1}+\alpha \gamma+\omega \gamma^{2}\right) \\
& -\frac{a_{2}}{4}\left[\beta^{2} \delta^{-1}+2 \beta \gamma+\delta \gamma \omega+2 \nu(\gamma-\omega)\right]\left(1+a_{1}\right)(1+\eta) \varepsilon_{\mathscr{L}}^{2} .
\end{aligned}
$$

Очевидно, что в условиях теоремы 6.2 параметры $0<\omega \leqslant \gamma / 3$ и $\eta>0$ можно подобрать так, чтобы $b \geqslant 0$. Поэтому (6.19) позволяет доказать, что из (6.15) вытекает (6.16). Таким образом, теорема 6.2 доказана.

Отметим, что аналоги теорем 6.1 и 6.2 могут быть установлены и в более общей ситуации. Например, можно считать нелинейные слагаемые зависяшими от пространственной переменной $x$, а вместо лапласиана рассмотреть эллиптический оператор второго порядка обшего вида. Кроме того, теореме 6.2 можно придать абстрактную форму, подобную теореме 4.3 и относящуюся к уравнениям второго порядка по времени вида (5.1).

\section{СПИСОК ЛИТЕРАТУРЫ}

[1] Бабин А. В., Вишик М. И. Аттракторы эволюционных уравнений. М.: Наука, 1989.

[2] Temam R. Infinite-dimensional Dynamical Systems in Mechanics and Physics. New York: Springer-Verlag, 1988.

[3] Ладыженская О. А. О нахождении минимальных глобальных аттракторов для уравнения Навье-Стокса и других уравнений с частными производньми // УМН 1987. Т. 42. №6. C. $25-60$.

[4] Чуешов И. Д. Глобальные аттракторы в нелинейных задачах математической физики // УМH. 1993. T. 48. № 3. C. 135-162.

[5] Chepyzhov V.V., Vishik M. I. Attractors of non-autonomous dynamical systems and their dimension // J. Math. Pures Appl. 1994. V. 73. № 3. P. 279-333.

[6] Foias C., Prodi G. Sur le comportement global des solutions non stationnaires des équations de Navier-Stokes en dimension deux // Rend. Sem. Mat. Univ. Padova. 1967. V. 39. P. 1-34.

[7] Ладыженская О.А.О динамической системе, порождаемой уравнениями Навье-Стокса // Зап. научн. семин. ЛОМИ. 1972. Т. 27. С. 91-115.

[8] Foias C., Manley O.P., Temam R., Treve Y.M. Asymptotic analysis of the Navier-Stokes equations // Physica D. 1983. V. 9. P. 157-188.

[9] Sermange M., Temam R. Some mathematical questions related to MHD equations // Commun. Pure Appl. Math. 1983. V. 36. P. 635-664.

[10] Foias C., Temam R. Determination of the solutions of the Navier-Stokes equations by a set of nodal values // Math. Comput. 1984. V. 43. P. 117-133.

[11] Ладыженская О.А. Об оценках фрактальной размерности и числа определяющих мод для инвариантных множеств динамических систем // Зап. научн. семин. ЛОМИ. 1987. T. 163. C. $105-129$.

[12] Foias C., Titi E.S. Determining nodes, finite difference schemes and inertial manifolds // Nonlinearity. 1991. V. 4. №1. P. 135-153.

[13] Jones D. A., Titi E.S. Determination of the solutions of the Navier-Stokes equations by finite volume elements // Physica D. 1992. V. 60. P. 165-174.

[14] Jones D.A., Titi E.S. Upper bounds on the number of determining modes, nodes and volume elements for the Navier-Stokes equations // Indiana Univ. Math. J. 1993. V. 42. № 3. P. 875-887.

[15] Kukavica I. On the number of determining nodes for the Ginzburg-Landau equation // Nonlinearity. 1992. V. 5. № 5. P. 997-1006.

[16] Foias C., Kukavica I. Determining nodes for the Kuramoto-Sivashinsky equation // J. Dyn. Diff. Eq. 1995. V. 7. №2. P. 365-373. 
[17] Cockburn B., Jones D. A., Titi E.S. Determining degrees of freedom for nonlinear dissipative systems // C. R. Acad. Sci. Paris. Ser. 1. 1995. V. 321. P. 563-568.

[18] Чуешов И. Д. О функционалах, однозначно определяющих асимптотическое поведение решений системы Кармана // УМН. 1996. Т. 51. № 5. С. 162.

[19] Bernier-Kazantsev C., Chueshov I. D. The finiteness of determining degrees of freedom for the quasi-geostrophic multi-layer ocean model // Preprint №3067. Nancy: INRIA-Lorraine, 1996.

[20] Чуешов И.Д. Замечание о множествах определяющих элементов для систем реакции-диффузии // Матем. заметки. 1998. Т. 63. № 5. С. 774-784.

[21] Chueshov I.D. On the finiteness of the number of determining elements for von Karman evolution equations // Math. Meth. Appl. Sci. 1997. V. 20. P. 855-865.

[22] Бабин А.В. Асимптотика при $|x| \rightarrow \infty$ функций, лежащих на аттракторе двумерной системш Навье-Стокса в неограниченной плоской области // Матем. сб. 1991. Т. 182. № 12. C. $1683-1709$.

[23] Foias C., Sell G. R., Temam R. Inertial manifolds for nonlinear evolutionary equations // J. Diff. Equat. 1988. V. 73. P. 309-353.

[24] Chow S.-N., Lu K. Invariant manifolds for flows in Banach spaces // J. Diff. Equat. 1988. V. 74. P. $285-317$.

[25] Constantin P., Foias C., Nicolaenko B., Temam R. Integral Manifolds and Inertial Manifolds for Dissipative Partial Differential Equations. New York: Springer-Verlag, 1989.

[26] Чуешов И. Д. Введение в теорию инерциальных многообразий. Харьков: Изд-во Харьк. ун-та, 1992.

[27] Рудин У. Функциональный анализ. М.: Мир, 1975.

[28] Тихомиров В. М. Некоторые вопросы теории приближений. М.: Изд-во МГУ, 1976.

[29] Бабенко К. И. Основы численного анализа. М.: Наука, 1986.

[30] Aubin J.-P. Approximation of Elliptic Boundary-Value Problems. New York: Wiley, 1972.

[31] Трибель Х. Теория интерполяции, функциональные пространства, диффференциальные операторы.. М.: Мир, 1980.

[32] Гохберг И. Ц., Крейн М. Г. Введение в теорию линейных несамосопряженных операторов. М.: Наука, 1965.

[33] Мазья В. Г. Пространства С. Л. Соболева. Л.: Изд-во ЛГУ, 1985.

[34] Лионс ЖК.-Л., Мадженес Э. Неоднородные граничные задачи и их приложения. М.: Мир, 1971.

[35] Ладыженская О.А. Математические вопросы динамики вязкой несжимаемой жидкости. М.: Наука, 1970.

[36] Constantin P., Foias C. Navier-Stokes Equations. Chicago: Univ. of Chicago Press, 1988.

[37] Hassard B. D., Kazarinoff N. D., Wan Y.-H. Theory and Application of Hopf Bifurcation. Cambridge: Cambridge Univ. Press, 1981.

[38] Henry D. Geometric Theory of Semilinear Parabolic Equations. Berlin: Springer-Verlag, 1981.

[39] Smoller J. Schock Waves and Reaction-Diffusion Equations. New York: Springer-Verlag, 1983.

[40] Marion M. Inertial manifolds associated to partly dissipative reaction-diffusion systems // J. Math. Anal. Appl. 1989. V. 143. P. 295-326.

[41] Promislow K. Time analy ticity and Gevrey regularity for solutions of a class of dissipative partial differential equations // Nonlinear Anal. Theory Methods Appl. 1991. V. 16. № 11. P. 959-980.

[42] Duan J., Titi E., Holmes P. Regularity, approximation and asymptotic dynamics for a generalized Ginzburg-Landau equation // Nonlinearity. 1993. V. 6. № 6. P. 915-933.

[43] Чуешов И.Д. Конечномерность аттрактора в некоторых задачах нелинейной теории оболочек // Матем. сб. 1987. Т. 133 (175). № 4. C. 419-428.

[44] Chueshov I. D. On a construction of approximateinertial manifolds for second order in time evolution equations // Nonlinear Anal. Theory Methods Appl. 1996. V. 26. P. 1007-1021.

[45] Hale J.K. Theory of Functional Differential Equations. New York: Springer-Verlag, 1977. 
[46] Чуешов И. Д. Об одной системе уравнений с запаздьванием, возникающей в аэроупругости // Теория функций, функц. анал. и их прил. 1990. № 54. С. 123-130.

[47] Boutet de Monvel L., Chueshov I. D. Non-linear oscillations of a plate in a flow of gas // C. R. Acad. Sci. Paris. Ser. I. 1996. V. 322. № 10. P. 1001-1006.

[48] Chueshov I. D., Rezounenko A. V. Global attractors for a class of retarded quasilinear partial differential equations // C. R. Acad. Sci. Paris. Ser. I. 1995. V. 321. № 5. P. 607-612.

[49] Ладыженская О.А., Солонников В.А., Уральцева Н. Н. Линейные и квазилинейные уравнения параболического типа. М.: Наука, 1967.

[50] Lasiecka I., Tataru D. Uniform boundary stabilization of semilinear wave equations with nonlinear boundary damping // Diff. Int. Equat. 1993. V. 6. P. 507-533.

Харьковский университет, Украина

Поступила в редакцию

15.09.1997 